\title{
Evidence For Non-Linear Causality Between Public Spending And Income In The European Union Countries
}

Stella Karagianni, University of Macedonia, Greece

Maria Pempetzoglou, Democritus University of Thrace, Greece

\begin{abstract}
This paper deploys the non-linear Granger causality methods in order to determine the causal relationship between national income and government expenditure in the European Union countries over the post-war time period. For this purpose, six alternative functional forms of Wagner's law have been adopted. The empirical results indicate support for non-linear causality between income and government expenditure and they may prove useful theoretical and empirical research for the regulators and the policy makers.
\end{abstract}

Keywords: > non-linear Granger causality, Wagner's law, EU countries

\section{INTRODUCTION}

¿ $\mathrm{n}$ the 1880s, the German economist Adolph Wagner analyzed the trends in the growth of public expenditure relatively to the size of the public sector. His observations led to what is now called "Wagner's law" or the "law of rising public expenditure" [Musgrave et al. (1989), Trotman-Dickenson (1996)], according to which the rise in public expenditure will be more than proportional to the increase in the national income and will thus result in a relative expansion of the public sector. This law has been the subject of extensive investigations, especially during the latest decades, as the development of modern industrial society is anticipated to have given rise to increasing political "pressure for social progress" and calls for increased allowance for "social consideration".

In their attempt to examine the existence of a short run relationship between government expenditure and economic development as well as to determine the causal flow running between these two variables, researchers carried on linear causality tests. All studies on causal relationships rely exclusively on traditional linear Granger causality tests [Sahni and Singh (1984a and 1984b), Chow et al. (2002), Chletsos and Kollias (1997), Islam (2001), Chang, (2002), Osaretin et al. (2004), Loizides et al. (2005), Karagianni et al. (2002), Karagianni (2000), etc.].

However, linear tests alone provide weak and limited conclusions. Baek and Brock (1992) argue that linear causality tests can be weak in detecting non-linear causal relations. Recent advances in econometrics allow the use of non-linear causality techniques to test for the directions and causal flows between government spending and income. Non-linear causality tests are considered as specification tools for uncovering significant non-linearities in the dynamic interrelationships between economic variables (Hiemstra and Jones, 1994). A study that has been carried out using the non-linear Granger causality test was conducted by Karagianni et al. (2003) and it investigated the directions and patterns of non-linear causality between government spending and gross domestic product in the Greek economy. The empirical results indicated support for non-linear causality between national income and government expenditure in six alternative functional forms of Wagner's law.

The aim of this paper is to investigate Wagner's law hypothesis by applying the non-linear Granger causality test in the European Union countries over the post-war time period. The non-linear Granger causality approach is expected to provide additional empirical evidence of Wagner's law in the European Union economies. 
The implementation the non-linear Granger causality test, which is considered to be one of the most advanced econometric methods, is the innovative feature of this study. The purpose of testing Wagner's law for any non-linear causality is that it may highlight an additional avenue for which a link between government spending and income may exist and be hitherto undetected. If it is observed that non-linear causality exists, that it would imply a far more complex relationship exists between these two variables than has previously been documented. Moreover, Granger (1989) argues that uni-variate and multi-variate non-linear models represent the proper way to model a real world that is almost certainly non-linear.

The remainder of the paper is organized as follows. Section 2 analyses the alternative functional forms of Wagner's law. Section 3 provides an overview of the empirical methodology. Section 4 presents the data and the empirical findings of the relationship between government expenditure and economic development. Section 5 concludes and section 6 gives suggestions for future research.

\section{ALTERNATIVE FUNCTIONAL FORMS OF WAGNER'S LAW}

The initial idea of Wagner's law, where the public sector size is assumed to be a function of economic development, has raised strong disagreements among researchers about the precise formulation of the law. In this paper, we aim to investigate the nature of the relationships between government spending and national income and, for this purpose, six alternative functional forms of the law are being examined ${ }^{1}$ :

\begin{tabular}{|c|c|}
\hline$(E)_{t}=A(G D P)_{t}^{\beta}$ & (I) \\
\hline$(C)_{t}=A(Y)_{t}^{\beta}$ & $(I I)$ \\
\hline$(E)_{t}=A\left(\frac{G D P}{N}\right)_{t}^{\beta}$ & (III) \\
\hline$\left(\frac{E}{G D P}\right)_{t}=A\left(\frac{G D P}{N}\right)_{t}^{\beta}$ & $(I V)$ \\
\hline$\left(\frac{E}{N}\right)_{t}=A\left(\frac{G D P}{N}\right)_{t}^{\beta}$ & $(V)$ \\
\hline$\left(\frac{E}{G D P}\right)_{t}=A(G D P)_{t}^{\beta}$ & $(V I)$ \\
\hline
\end{tabular}

where E stands for government expenditure, GDP stands for gross domestic product, C stands for government consumption, $\mathrm{Y}$ for national income, $\mathrm{N}$ for the population.

The first formulation was adopted by Peacock and Wiseman [1961], who interpreted the law as follows: "public expenditures should increase by a higher rate than GDP". The second formulation was created by Pryor [1968], who stated that "in developing countries, the share of public consumption expenditure to the national income is increasing. In the same year, Goffman [1968] expressed the law in a different way: "during the development process, the GDP per capita increase should be lower than the rate of public sector activities increase". According to Musgrave [1969], in the fourth equation, "the public sector share to GDP is increasing as the GDP per capita raises, during the development process". Gupta [1967] considered per capita government expenditure as a function of per capita GDP (fifth equation). At last, Mann [1980], in his attempt to verify empirically the existence of Wagner's law, adopted the sixth formulation, according to which "public expenditure share to GDP is a function of GDP”. 


\section{METHODOLOGY}

In this section, the definitions of the non-linear Granger causality tests are discussed. At this part, a presentation of the statistical technique - developed by Baek and Brock (1992) and modified by Hiemstra and Jones (1994) - used to test for non-linear Granger causality - is been made.

\section{The Non-Linear Granger Causality test}

\section{Baek and Brock test}

Baek and Brock (1992) propose a non-parametric statistical method for detecting non-linear causal relations that cannot be uncovered by equivalent linear tests. Their approach ${ }^{2}$ employs the correlation integral, which provides an estimate of spatial dependence across time. ${ }^{3}$ Consider two stationary and weakly dependent time series $\left\{X_{\mathrm{t}}\right\}$ and $\left\{Y_{\mathrm{t}}\right\}, \mathrm{t}=1,2, \ldots, \mathrm{n}$. Let the $m$-length lead vector $\mathrm{X}_{\mathrm{t}}$ be designated by $\mathrm{X}_{\mathrm{t}}{ }^{\mathrm{m}}$, and the $L \mathrm{X}$-length and the $L \mathrm{y}$ length lag vectors of $X_{\mathrm{t}}$ and $Y_{\mathrm{t}}$ be designated by $\mathrm{X}^{\mathrm{Lx}}{ }_{\mathrm{t}-\mathrm{Lx}}$ and $\mathrm{Y}^{\mathrm{Ly}}{ }_{\mathrm{t}-\mathrm{Ly}}$, respectively.

For given values of $m, L x$, and $L y \geq 1$ and for $e>0$, Y does not strictly Granger cause $\mathrm{X}$ if:

$$
\begin{array}{r}
\operatorname{Pr}\left(\left\|X_{t}^{m}-X_{s}^{m}\right\|<e \mid\left\|X_{t-L x}^{L x}-X^{L x}{ }_{s-L x}\right\|<e,\left\|Y^{L y}{ }_{t-L y}-Y^{L y}{ }_{s-L y}\right\|<e\right) \\
=\operatorname{Pr}\left(\left\|X_{t}^{m}-X_{s}^{m}\right\|<e \mid\left\|X^{L x}{ }_{t-L x}-X^{L x}{ }_{s-L x}\right\|<e\right),
\end{array}
$$

where $\operatorname{Pr}($.$) denotes probability and \|$.$\| denotes the maximum norm (the maximum norm for Z \equiv\left(Z_{1}, Z_{2}, \ldots, Z_{K}\right)$ is defined as the $\max \left(\mathrm{Z}_{\mathrm{i}}\right), \mathrm{i}=1,2, \ldots, \mathrm{K}$

The probability on the left hand side of the above equation is the conditional probability that the two arbitrary $m$-length lead vectors $\left\{\mathrm{X}_{\mathrm{t}}\right\}$ are within a distance $e$ of each other, given that the corresponding $\mathrm{L}_{\mathrm{x}}$-length lag vectors of $\left\{\mathrm{X}_{\mathrm{t}}\right\}$ and $\mathrm{L}_{\mathrm{y}}$-length lag vectors of $\left\{\mathrm{Y}_{\mathrm{t}}\right\}$ are within $e$ of each other. The probability on the right hand side of the equation is the conditional probability that two arbitrary $m$-length lead vectors of $\left\{\mathrm{X}_{\mathrm{t}}\right\}$ are within a distance $e$ of each other, given that their corresponding $\mathrm{L}_{\mathrm{x}}$-length lag vectors are within a distance $e$ of each other.

In order to test for non-linear Granger causality, we need first to remove the linear dependence. For this reason we apply a Vector Autoregression (VAR) model and use the estimate residuals to test for non-linear causality. We estimate the following VAR model -the VAR model filters structures and does not remove trends- for each country $\mathrm{i}$, where $\varepsilon_{\mathrm{i}, \mathrm{t}}$ is the innovation at time $\mathrm{t}$ and $\mathrm{p}$ the lag length:

$$
\begin{aligned}
& \mathrm{GE}_{\mathrm{i}, \mathrm{t}}=\sum_{k=1}^{p} \delta_{i, k} G E_{i, t-k}+\sum_{k=1}^{p} \beta_{i, k} \mathrm{ED}_{\mathrm{i}, \mathrm{t}-\mathrm{k}}+\varepsilon_{\mathrm{i}, \mathrm{t}} \\
& \mathrm{ED}_{\mathrm{i}, \mathrm{t}}=\sum_{k=1}^{p} \delta_{i, k} G E_{i, t-k}+\sum_{k=1}^{p} \beta_{i, k} \mathrm{ED}_{\mathrm{i}, \mathrm{t}-\mathrm{k}}+\mathrm{u}_{\mathrm{i}, \mathrm{t}}
\end{aligned}
$$

The strict Granger non-causality condition in the VAR model can be written as:

$$
\frac{\mathrm{CI}_{1}\left(\mathrm{~m}+\mathrm{L}_{\mathrm{x}}, \mathrm{L}_{\mathrm{y},} \mathrm{e}\right)}{\mathrm{CI}_{2}\left(\mathrm{~L}_{\mathrm{x}}, \mathrm{L}_{\mathrm{y}}, \mathrm{e}\right)}=\frac{\mathrm{CI}_{3}\left(\mathrm{~m}+\mathrm{L}_{\mathrm{x}}, \mathrm{e}\right)}{\mathrm{CI}_{4}\left(\mathrm{~L}_{\mathrm{x}}, \mathrm{e}\right)}
$$

$C I_{1}, C I_{2}, C I_{3}$ and $C I_{4}$ in the above equation are the correlation-integral estimators of the joint probabilities, which are discussed in detail by Hiemstra and Jones (1994). For given values of $m, L x$ and $L y \geq 1$ and 
for $e>0$ under the assumptions that $\left\{\mathrm{X}_{\mathrm{t}}\right\}$ and $\left\{\mathrm{Y}_{\mathrm{t}}\right\}$ are strictly stationary, weakly dependent and satisfy the mixing conditions of Denker and Keller (1983), if $\left\{Y_{t}\right\}$ does not strictly Granger cause $\left\{X_{t}\right\}$ then,

$$
\sqrt{\mathrm{n}}\left[\frac{\mathrm{CI}_{1}\left(\mathrm{~m}+\mathrm{L}_{\mathrm{X}}, \mathrm{L}_{\mathrm{y}}, \mathrm{e}, \mathrm{n}\right)}{\mathrm{CI}_{2}\left(\mathrm{~L}_{\mathrm{x}}, \mathrm{L}_{\mathrm{y}}, \mathrm{e}, \mathrm{n}\right)}=\frac{\mathrm{CI}_{3}\left(\mathrm{~m}+\mathrm{L}_{\mathrm{X}}, \mathrm{e}, \mathrm{n}\right)}{\mathrm{CI}_{4}\left(\mathrm{~L}_{\mathrm{X}}, \mathrm{e}, \mathrm{n}\right)}\right] \sim \mathrm{N}\left(0, \sigma^{2}(m, L x, L y, e)\right.
$$

A significantly positive test statistic in the above equation suggests that lagged values of $\mathrm{Y}$ help to predict $\mathrm{X}$, whereas a significant negative value suggests that knowledge of the lagged values of $\mathrm{Y}$ confounds the prediction of X. For this reason, Hiemstra and Jones (1994) argue that the test statistic in the above equation should be evaluated with right-tailed critical values when testing for the presence of Granger causality. In order to test for nonlinear Granger causality the above test is applied to the two estimated residual series from the VAR models.

\section{Modified Baek and Brock test}

Baek and Brock's version of the test has been applied by Hiemstra and Jones (1994) and is based on the assumption of mutually independent and individually iid for the errors of the maintained VAR model. The modified test holds under the more general case where the errors are allowed to be weakly dependent. The fundamental difference between the two versions of the test occurs in the estimators of $\sigma^{2}(m, L x, L y, e)$ in the last equation.

\section{DATA AND EMPIRICAL RESULTS}

\subsection{Data}

The present analysis has been carried out using annual data for the European Union countries for the period 1949-1998. The data is obtained from various volumes of the International Financial Statistics. Expenditure comprises all non-repayable payments by government, whether requited or unrequited and whether for current or capital purposes. Gross Domestic Product (GDP) is the sum of final expenditures: exports of goods and services, imports of goods and services, private consumption, government consumption, gross fixed capital formation and increase/decrease in stocks. Adding net factor income/payments abroad to GDP produces Gross National Income, formerly known as Gross National Product.

\subsection{Results of the Non-Linear Granger Causality Test}

To implement the modified Baek and Brock (1992) test, as proposed by Hiemstra and Jones (1994), a choice of values for the lead length, $m$, the lag lengths $L x$ and $L y$, and the scale parameter, $e$, must be made. In this study, we have set the lead length at $m=1$ and $L x=L y$ and we have used common lag length of 1 to 10 lags. Table 1 indicates the number of lags that have been used in each equation for every country. This study also uses common scale parameter of $e=1.5 \sigma$, where $\sigma$ denotes the standard deviation of the standardized time series.

The empirical results indicate some individual cases of bi-directional non-linear causality flows. We should mention that bi-directional non-linear causality refers to non-proportional feedback relationships between variables. The presence of non-linearity in the comovements of economic variables decreases predictability and raises sensitivity of variables responses to economic shocks. A bi-directional flow indicates that both national income effects government expenses and vice versa and it is observed in equations I and III in Belgium and Greece, equation II in Finland and Portugal, III in Ireland, IV and V in Italy and equation VI in the Netherlands. These findings denote that a shock in both government expenditures and gross national product is expected to affect in a non-proportional way national income and government spending, respectively, due to the existence of the non-linear causality. 
Table 1: Number of lags

\begin{tabular}{|c|c|c|c|c|c|c|}
\hline \multirow[b]{2}{*}{ Countries } & \multicolumn{6}{|c|}{ Equations } \\
\hline & I & II & III & IV & $\mathbf{V}$ & VI \\
\hline Austria & 4 & 3 & 4 & 4 & 4 & 4 \\
\hline Belgium & 1 & 1 & 1 & 1 & 1 & 1 \\
\hline Denmark & 1 & 3 & 1 & 1 & 1 & 1 \\
\hline Finland & 3 & 2 & 3 & 3 & 3 & 3 \\
\hline France & 1 & 1 & 1 & 1 & 1 & 1 \\
\hline Germany & 1 & 1 & 1 & 1 & 1 & 1 \\
\hline Greece & 1 & 2 & 1 & 1 & 2 & 1 \\
\hline Ireland & 1 & 2 & 2 & 2 & 2 & 1 \\
\hline Italy & 1 & 2 & 1 & 1 & 1 & 1 \\
\hline Luxembourg & 2 & 2 & 2 & 2 & 2 & 2 \\
\hline Netherlands & 1 & 1 & 1 & 1 & 1 & 1 \\
\hline Portugal & 1 & 1 & 1 & 1 & 1 & 1 \\
\hline Spain & 1 & 1 & 1 & 1 & 1 & 1 \\
\hline Sweden & 3 & 1 & 3 & 3 & 3 & 3 \\
\hline U.K. & 1 & 1 & 1 & 1 & 1 & 1 \\
\hline
\end{tabular}

Table 2 presents a summary view of the empirical results of the non-linear Granger causality test, while the analytical results are reported in Appendix.

Table 2: Non-Linear Granger Causality Test

\begin{tabular}{|c|c|c|c|c|c|c|}
\hline \multirow[b]{2}{*}{ Countries } & \multicolumn{6}{|c|}{ Equations } \\
\hline & I & II & III & IV & V & VI \\
\hline Austria & $\varnothing$ & $\varnothing$ & $\varnothing$ & $\varnothing$ & $\varnothing$ & $\varnothing$ \\
\hline Belgium & $\Leftrightarrow$ & $\varnothing$ & $\Leftrightarrow$ & $\diamond$ & كم & كڤ \\
\hline Denmark & $\Rightarrow$ & $\Rightarrow$ & $\Rightarrow$ & $\Rightarrow$ & $\Rightarrow$ & $\Rightarrow$ \\
\hline Finland & $\Rightarrow$ & $\Leftrightarrow$ & $\Rightarrow$ & $\varnothing$ & $\Rightarrow$ & $\varnothing$ \\
\hline France & $\varnothing$ & $\varnothing$ & $\varnothing$ & $\varnothing$ & $\varnothing$ & $\varnothing$ \\
\hline Germany & $\varnothing$ & $\varnothing$ & $\varnothing$ & $\varnothing$ & $\varnothing$ & $\varnothing$ \\
\hline Greece & $\Leftrightarrow$ & $\Rightarrow$ & $\Leftrightarrow$ & $\varnothing$ & $\varnothing$ & $\varnothing$ \\
\hline Ireland & ڤ & $\varnothing$ & $\Leftrightarrow$ & $\varnothing$ & $\varnothing$ & $\varnothing$ \\
\hline Italy & $\varnothing$ & $\varnothing$ & ڤ & $\Leftrightarrow$ & $\Leftrightarrow$ & $\varnothing$ \\
\hline Luxembourg & ڤ & $\varnothing$ & $\Rightarrow$ & ڤ & $\varnothing$ & كـ \\
\hline Netherlands & $\varnothing$ & $\Rightarrow$ & $\varnothing$ & $\Rightarrow$ & $\Rightarrow$ & $\Leftrightarrow$ \\
\hline Portugal & $\varnothing$ & $\Leftrightarrow$ & $\Rightarrow$ & $\varnothing$ & $\varnothing$ & $\varnothing$ \\
\hline Spain & $\varnothing$ & ڤ & $\varnothing$ & ڤ & $\varnothing$ & $\varnothing$ \\
\hline Sweden & ڤ & $\varnothing$ & $\varnothing$ & $\varnothing$ & $\varnothing$ & هـ \\
\hline U.K. & ڤ & $\Rightarrow$ & ڤ & $\varnothing$ & كم & $\varnothing$ \\
\hline
\end{tabular}

$\Leftrightarrow$ non-linear Granger causality exists on both directions

$\Rightarrow$ non-linear Granger causality exists only from the dependent towards the independent variable

$\diamond$ non-linear Granger causality exists only from the independent towards the dependent variable

$\varnothing$ No non-linear Granger causality exists

Signs of non-linear causality are not observed at all in all equations in Austria, France and Germany, in equation II in Belgium, IV in Finland, IV, V and VI in Greece, II, IV, V and VI in Ireland, I, II and VI in Italy, II and V in Luxembourg, I and III in the Netherlands, I, IV, V and VI in Portugal, I, III, V and VI in Spain, II, III, IV and V in Sweden and IV and VI in the UK. This is due to the fact that either non-linear flows cannot be detected or no such flows exist in these specific set of countries and equations. 
Non-linear uni-directional flows indicate that either national income affects government expenses or government spending affects income and they are observed in the remaining equations.

More specifically, in some cases - in all equations in Denmark, in equations I, III and V in Finland, II in Greece and the UK, III in Luxembourg and Portugal and II, IV and V in the Netherlands - we observe only a unidirectional non-linear flow running from the dependent towards the independent variable. This fact indicates that a shock in government expenditures is expected to have disproportionate effects on national income and policy makers cannot detect the results of a shock they may cause in government expenditures on the gross domestic product.

In the rest of the cases - in equations IV, V and VI in Belgium, in equation I in Ireland, III in Italy, I, IV and VI in Luxembourg, II and IV in Spain, I and VI in Sweden and I, III and V in the UK - a unidirectional nonlinear flow is running from the independent towards the dependent variable. This indicates that a shock in national income is expected to have disproportionate effects on government spending and policy makers are unable to know ex ante the results of a shock they may cause in an economy.

\section{CONCLUSION}

In this paper, we have tried to determine the existence of non-linear causality between government expenditure and national income in the European Union countries. The non-linear Granger causality test is implemented in six alternative functional interpretations of Wagner's law for the time-period 1949-1998.

The empirical findings indicate signs of non-linear causality but suggest that patterns of causality between income and government expenditure display dramatic differences across various countries. Some equations present a bi-directional flow of non-linear causality. This fact indicates that a shock in government expenditures is expected to affect national income in a non-proportional way and vice-versa. In these cases, the structural relationship seems to be strong.

In other equations in specific countries, non-linear flows are not observed at all. This may be due to the fact that either non-linear flows cannot be detected or no such flows exist.

In some cases, we observe unidirectional non-linear flows running either from the dependent towards the independent variable or from the independent towards the dependent variable. These findings indicate that a shock in each variable is expected to have disproportionate effects on the other variable and policy makers cannot detect the results that a shock - they may cause - in a variable is bound to have on the other variable.

Overall, the results contribute to the empirical literature by indicating the presence of non-linear causality between national income and public spending in some European Union countries. Non-linear results are better and more robust and the results they provide are more proper and advanced. The findings may prove useful to future theoretical and empirical research on Wagner's law.

The purpose of the paper is to provide policy makers with correct information and clearest signals as far as the potential forms of causality between national accounts' variables is concerned and to 'warn' them to pay attention to the shocks they intend to raise in an economy, since they do not know how a modification (increase or decrease) in a component of government expenditure is going to affect national income. The existence of nonlinearity cannot provide clearly assessed results as far as the magnitude of the generated change is concerned. Thus, policy makers become unable to forecast the exact size of a variable change due to a policy they implement. Additionally, researchers should consider nonlinear theoretical mechanisms and empirical regularities when evaluating models of the joint dynamics of public expenditures and national income.

Non-linear causality also reflects the heterogenous behaviour of political parties and individuals as far as the level of government expenditures is concerned. Since the beginning of 80s, European governments - in their attempt to achieve both stability and economic development - tend to implement restrictive public policies, which 
they occasionally recall due to social pressure for public expenditure increase. In pre-election periods, there is a tendency for government expenditures to increase, while in the rest of the time governments insist on implementing restrictive public policies. The result of these contradictory policies is yet unknown, since it may also be influenced by electoral fluctuations.

\section{SUGGESTIONS FOR FUTURE RESEARCH}

The empirical results of this study can serve as a useful guide for further research. Future research can be pursued by disaggregating national accounts' data and by examining non-linear causality in each component of government expenditures. It is anticipated that each component of public expenditure is linked to the national income with either a linear or a non-linear relation. If non-linear causality relations are observed, policy makers should be cautious with the shocks they intend to raise in each component of government expenditure. 


\section{APPENDIX}

Results of the Non-Linear Granger Causality test

Non-Linear Granger Causality Test - AUSTRIA

\begin{tabular}{|c|c|c|c|c|c|c|c|c|c|c|c|c|c|c|c|c|c|c|c|c|c|c|c|c|}
\hline \multirow[b]{2}{*}{$x=L y$} & \multicolumn{2}{|c|}{ E-GDP } & \multicolumn{2}{|c|}{ GDP-E } & \multicolumn{2}{|c|}{$\mathrm{C}-\mathrm{Y}$} & \multicolumn{2}{|c|}{$\mathrm{Y}-\mathrm{C}$} & \multicolumn{2}{|c|}{ E-GDP/N } & \multicolumn{2}{|c|}{ GDP/N-E } & \multicolumn{2}{|c|}{ E/GDP-GDP/N } & \multicolumn{2}{|c|}{ GDP/N-E/GDP } & \multicolumn{2}{|c|}{ E/N-GDP/N } & \multicolumn{2}{|c|}{ GDP/N-E/N } & \multicolumn{2}{|c|}{ E/GDP-GDP } & \multicolumn{2}{|c|}{ GDP-E/GDP } \\
\hline & Cs & T-Val & Cs & $\mathrm{T}-\mathrm{V}$ & Cs & T- & Cs & T-I & Cs & T-Val & Cs & $\mathrm{T}-\mathrm{Va}$ & Cs & T-Val & Cs & T-Val & Cs & T-Val & Cs & T-Val & Cs & T-Val & Cs & T-Val \\
\hline 1 & 0.002 & 316 & -0.002 & \begin{tabular}{|l|l}
-0.783 \\
\end{tabular} & 0.004 & & -0.004 & -1.062 & 0.002 & & -0.002 & & & & .006 & & & & -0.002 & & .008 & & .004 & 578 \\
\hline 2 & -0.0 & 0 & & & & & & -1.051 & 2 & & & & & & & & & & & & & & & 879 \\
\hline 3 & -0.008 & 27 & & 103 & & & -0.006 & -1.05 & & & & & & & & & & & & & & & & 933 \\
\hline 4 & & & & & & & & -0.635 & & & & & & & & & & & & & & & & \\
\hline 5 & -0 & & & & & & -0.1 & -0.59 & & & & & & & & & & & & & & 746 & & 356 \\
\hline 6 & -0.0 & & & & & 32 & & -0.089 & & & & 561 & & & & & & & & 63 & & 754 & 33 & 263 \\
\hline 7 & -0. & & & & & (1) & & 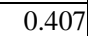 & & 32 & & 1.53 & & & & 94 & & & & 37 & & 338 & & .095 \\
\hline 8 & & & & & & & & & & & & & & & & & & & & & & & & 051 \\
\hline 9 & & & & & & & & 0.548 & & -1.2 & & & & & & & & & & & & 0.4 & & . \\
\hline 10 & -0.024 & \begin{tabular}{|l|}
-0.879 \\
\end{tabular} & 0.049 & 1.535 & 0.015 & 0.986 & 0.007 & 0.555 & -0.033 & -1.104 & 0.048 & 1.718 & 0.015 & 0.89 & .045 & 1.472 & -0.034 & -1.105 & & & 0.022 & 0.876 & 0.039 & 1.00 \\
\hline
\end{tabular}

Non-Linear Granger Causality Test - BELGIUM

\begin{tabular}{|c|c|c|c|c|c|c|c|c|c|c|c|c|c|c|c|c|c|c|c|c|c|c|c|c|}
\hline \multirow[b]{2}{*}{$\mathrm{Lx}=\mathrm{Ly}$} & \multicolumn{2}{|c|}{ E-GDP } & \multicolumn{2}{|c|}{ GDP-E } & \multicolumn{2}{|c|}{$\mathrm{C}-\mathrm{Y}$} & \multicolumn{2}{|c|}{$\mathrm{Y}-\mathrm{C}$} & \multicolumn{2}{|c|}{ E-GDP/N } & \multicolumn{2}{|c|}{ GDP/N-E } & \multicolumn{2}{|c|}{ E/GDP-GDP/N } & \multicolumn{2}{|c|}{ GDP/N-E/GDP } & \multicolumn{2}{|c|}{ E/N-GDP/N } & \multicolumn{2}{|c|}{ GDP/N-E/N } & \multicolumn{2}{|c|}{ E/GDP-GDP } & \multicolumn{2}{|c|}{ GDP-E/GDP } \\
\hline & Cs & $\mathrm{T}-\mathrm{Val}$ & Cs & $\mathrm{T}-\mathrm{Val}$ & Cs & T-Val & Cs & T-Val & Cs & $\mathrm{T}-\mathrm{Val}$ & Cs & $\mathrm{T}-\mathrm{Val}$ & Cs & T-Val & Cs & T-Val & Cs & T-Val & Cs & T-Val & Cs & $\mathrm{T}-\mathrm{Val}$ & Cs & T-Val \\
\hline 1 & 0.014 & 1.1 & 0.021 & $2.16^{*}$ & -0.021 & \begin{tabular}{|l|}
-1.008 \\
\end{tabular} & 0.015 & 0.809 & 0.024 & 1.693 & 0.016 & 1.711 & \begin{tabular}{|c|}
-0.004 \\
\end{tabular} & -0.154 & 0.016 & 1.542 & 0.022 & 1.509 & 0.018 & 1.826 & -0.011 & \begin{tabular}{|c|}
-0.477 \\
\end{tabular} & 0.021 & 1.857 \\
\hline 2 & -0.013 & -0.667 & -0.007 & -0.322 & -0.026 & \begin{tabular}{|l|}
-0.675 \\
\end{tabular} & 3E-04 & 0.006 & 0.009 & 0.419 & $-5 \mathrm{E}-04$ & -0.027 & \begin{tabular}{|l|}
-0.032 \\
\end{tabular} & -0.867 & 0.008 & 0.491 & -0.003 & -0.125 & 0.005 & 0.272 & -0.042 & -1.221 & 0.002 & 0.07 \\
\hline 3 & -0.056 & -1.789 & -0.005 & \begin{tabular}{|l|}
-0.144 \\
\end{tabular} & -0.064 & \begin{tabular}{|l|} 
\\
\end{tabular} & -0.029 & -0.53 & \begin{tabular}{|l|}
-0.035 \\
\end{tabular} & -1.039 & $-5 \mathrm{E}-04$ & -0.017 & \begin{tabular}{|l|}
-0.041 \\
\end{tabular} & -0.883 & 0.026 & 0.928 & -0.038 & \begin{tabular}{|l|}
-1.156 \\
\end{tabular} & 0.011 & 0.445 & -0.057 & -1.264 & .018 & 0.606 \\
\hline 4 & -0.105 & $-2.408 *$ & 0.006 & 0.113 & -0.011 & -0.107 & 0.033 & 0.351 & -0.064 & -1.377 & 0.017 & 0.377 & -0.014 & -0.292 & 0.074 & 1.946 & -0.065 & \begin{tabular}{|l|}
-1.547 \\
\end{tabular} & 0.039 & 0.993 & -0.049 & -0.988 & .055 & 1.263 \\
\hline 5 & -0.119 & $-2.859 *$ & 0.09 & 1.309 & 0.055 & 0.419 & 0.13 & 1.354 & -0.08 & $-2.026^{*}$ & 0.073 & 1.097 & -0.049 & -0.732 & 0.079 & 1.628 & -0.075 & -1.826 & 0.047 & 0.821 & -0.082 & -1.048 & 0.096 & 1.799 \\
\hline 6 & -0.12 & -1.065 & 0.063 & 0.579 & -0.111 & -0.595 & -0.086 & -0.468 & -0.096 & -0.89 & 0.072 & 0.702 & \begin{tabular}{|l|}
-0.027 \\
\end{tabular} & -0.237 & 0.072 & 0.801 & -0.086 & \begin{tabular}{|l|}
-0.832 \\
\end{tabular} & 0.017 & 0.156 & -0.046 & \begin{tabular}{|l|}
-0.383 \\
\end{tabular} & 0.063 & 0.657 \\
\hline 7 & -0.371 & -1.474 & 0.273 & 2.469* & & & & & \begin{tabular}{|l|}
-0.293 \\
\end{tabular} & -1.319 & 0.261 & $2.363 *$ & \begin{tabular}{|l|}
-0.184 \\
\end{tabular} & -0.84 & 0.261 & $2.363 *$ & -0.301 & -1.35 & 0.261 & $2.363 *$ & -0.256 & -1.024 & 0.273 & $2.469^{*}$ \\
\hline 8 & \begin{tabular}{|l|}
-0.387 \\
\end{tabular} & -1.346 & 0.278 & $2.253 *$ & & & & & \begin{tabular}{|l|}
-0.372 \\
\end{tabular} & $\begin{array}{l}-1.293 \\
\end{array}$ & \begin{tabular}{|c|}
0.278 \\
\end{tabular} & $2.253^{*}$ & \begin{tabular}{|c|}
-0.354 \\
\end{tabular} & -1.332 & 0.278 & $2.253 *$ & -0.389 & \begin{tabular}{|l|}
-1.353 \\
\end{tabular} & 0.278 & 2.253* & \begin{tabular}{|c|}
-0.354 \\
\end{tabular} & \begin{tabular}{|l|}
-1.332 \\
\end{tabular} & 0.278 & $2.253^{*}$ \\
\hline 9 & & & 0.267 & $2.352 *$ & & & & & & & 0.267 & $2.352 *$ & & & 0.267 & $2.352 *$ & & & 0.267 & $2.352 *$ & & & 0.267 & $2.352 *$ \\
\hline 10 & & & & & & & & & & & & & & & & & & & & & & & & \\
\hline
\end{tabular}

Non-Linear Granger Causality Test - DENMARK

\begin{tabular}{|c|c|c|c|c|c|c|c|c|c|c|c|c|c|c|c|c|c|c|c|c|c|c|c|c|}
\hline \multirow[b]{2}{*}{$\mathrm{Lx}=\mathrm{Ly}$} & \multicolumn{2}{|c|}{ E-GDP } & \multicolumn{2}{|c|}{ GDP-E } & \multicolumn{2}{|c|}{ C-Y } & \multicolumn{2}{|c|}{$\mathrm{Y}-\mathrm{C}$} & \multicolumn{2}{|c|}{ E-GDP/N } & \multicolumn{2}{|c|}{ GDP/N-E } & \multicolumn{2}{|c|}{ E/GDP-GDP/N } & \multicolumn{2}{|c|}{ GDP/N-E/GDP } & \multicolumn{2}{|c|}{ E/N-GDP/N } & \multicolumn{2}{|c|}{ GDP/N-E/N } & \multicolumn{2}{|c|}{ E/GDP-GDP } & \multicolumn{2}{|c|}{ GDP-E/GDP } \\
\hline & Cs & T-Val & $\mathrm{Cs}$ & $\overline{T-V}$ & Cs & T-Val & $\overline{s s}$ & $\mathrm{~T}-\mathrm{Val}$ & $\mathrm{Cs}$ & T-Val & Cs & $T-V_{i}$ & Cs & T-Val & Cs & & $\mathrm{Cs}$ & $\mathrm{T}-\mathrm{Val}$ & Cs & T-Val & $\mathrm{Cs}$ & T-Val & $\mathrm{Cs}$ & T-Val \\
\hline 1 & 0.036 & 1.293 & -0.018 & -0.837 & 0.027 & $2.369 *$ & & & & & & & & & \begin{tabular}{|l|}
-0.014 \\
\end{tabular} & & & & & & & & & \\
\hline 2 & & & & 1.241 & & & & & & & & & & & & & & & & & & & & 25 \\
\hline 3 & 0.04 & & 8 & 1.3 & 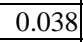 & & 2 & -0.0 & & & & & & & & & & & & & & & & \\
\hline 4 & 0 & 1. & 07 & -0.1 & 0.0 & & $-4 \mathrm{E}$ & $-0 .($ & 59 & & & -0.8 & & & & & & & & 99 & & & 01 & \\
\hline 5 & 0.095 & 1.259 & -0.045 & -0.537 & 0.033 & 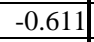 & -0.03 & -0.362 & 0.096 & 1.146 & -0.078 & -0.859 & -0.024 & -0.248 & & 451 & 22 & 91 & -0.028 & -0.302 & -0 & 0.396 & 0.009 & 0.12 \\
\hline 6 & 0.231 & $3.548 *$ & 0.056 & 0.541 & -0.048 & 47 & 11 & 0.089 & 0.28 & $6^{*}$ & & 0.249 & & 0.818 & & 225 & 317 & $51 *$ & & 622 & & 467 & .092 & 839 \\
\hline 7 & 0.231 & 2.147* & -0.042 & -0.337 & -0.058 & \begin{tabular}{|l|}
-0.445 \\
\end{tabular} & 0.01 & 0.077 & 0.261 & $2.309^{*}$ & -0.101 & -0.801 & 193 & 1.314 & 09 & 0.069 & 0.304 & $2.53 *$ & -0.075 & -0.568 & 86 & 0.479 & 0.1 & .842 \\
\hline 8 & 0.319 & 1.623 & -0.041 & -0.177 & 0.158 & 1.89 & -0.111 & -0.835 & 0.44 & $3.038^{*}$ & -0.039 & -0.162 & 356 & $3.295 *$ & & 0.05 & 0.48 & $3.465 *$ & 0.012 & 0.05 & 0.341 & $3.059 *$ & 0.109 & 0.458 \\
\hline 9 & 0.571 & 3.614* & -0.125 & -0.432 & & & & & 0.5 & $2.591 *$ & -0.275 & -0.859 & 286 & $3.359 *$ & \begin{tabular}{|l|}
-0.267 \\
\end{tabular} & 0.873 & 539 & $3.351 *$ & -0.267 & $\begin{array}{l}-0.873 \\
\end{array}$ & 286 & $3.359 *$ & -0.125 & -0.432 \\
\hline 10 & 0.777 & 5.571* & & & & & & & 0.714 & $3.913^{*}$ & & & 0.211 & $2.416^{*}$ & & & 0.714 & 3.913* & & & 0.211 & $2.416^{*}$ & & \\
\hline
\end{tabular}


Non-Linear Granger Causality Test - FINLAND

\begin{tabular}{|c|c|c|c|c|c|c|c|c|c|c|c|c|c|c|c|c|c|c|c|c|c|c|c|c|}
\hline \multirow[b]{2}{*}{$\mathrm{Lx}=\mathrm{Ly}$} & \multicolumn{2}{|c|}{ E-GDP } & \multicolumn{2}{|c|}{ GDP-E } & \multicolumn{2}{|c|}{$\mathrm{C}-\mathrm{Y}$} & \multicolumn{2}{|c|}{$\mathrm{Y}-\mathrm{C}$} & \multicolumn{2}{|c|}{ E-GDP/N } & \multicolumn{2}{|c|}{ GDP/N-E } & \multicolumn{2}{|c|}{ E/GDP-GDP/N } & \multicolumn{2}{|c|}{ GDP/N-E/GDP } & \multicolumn{2}{|c|}{ E/N-GDP/N } & \multicolumn{2}{|c|}{ GDP/N-E/N } & \multicolumn{2}{|c|}{ E/GDP-GDP } & \multicolumn{2}{|c|}{ GDP-E/GDP } \\
\hline & Cs & T-Val & Cs & $\mathrm{T}-\mathrm{Val}$ & Cs & $\mathrm{T}-\mathrm{Val}$ & Cs & $\mathrm{T}-\mathrm{Val}$ & Cs & $\mathrm{T}-\mathrm{Val}$ & Cs & $\mathrm{T}-\mathrm{Val}$ & Cs & T-Val & Cs & T-Val & Cs & T-Val & Cs & $\mathrm{T}-\mathrm{Val}$ & Cs & T-Val & Cs & T-Val \\
\hline 1 & 0.013 & 0.74 & 0.011 & 0.698 & -0.017 & -0.865 & -0.038 & -1.723 & 0.015 & 0.947 & 0.013 & 0.984 & 0.043 & 1.252 & 0.016 & .952 & 0.016 & 0.978 & 0.008 & 0.593 & 0.045 & 1.275 & .018 & 0.9 \\
\hline 2 & 0.038 & 1.26 & 0.026 & 0.894 & -0.04 & -0.934 & -0.049 & -0.952 & 0.032 & 1.131 & 0.033 & 1.179 & 0.037 & 1.012 & 0.043 & 1.19 & 0.037 & 1.294 & 0.033 & 1.214 & 0.037 & 1.012 & 0.043 & 1.111 \\
\hline 3 & 0.087 & $2.55^{*}$ & 0.016 & 0.368 & -0.137 & -1.459 & 0.013 & 0.168 & 0.087 & $2.568 *$ & 0.03 & 0.763 & 0.013 & 1.323 & 0.042 & 0.992 & 0. & $2.549 *$ & 0.039 & 0.941 & 13 & 1.323 & 37 & 0.876 \\
\hline 4 & 0.07 & 1.49 & -0.017 & -0.313 & -0.166 & -1.281 & 0.025 & 0.197 & 0.084 & $2.148 *$ & 0.003 & 0.062 & 0.009 & 984 & 0.017 & 0.401 & 0.084 & $2.087 *$ & 0.014 & 0.292 & 009 & 0.984 & .019 & 0.422 \\
\hline 5 & 0.078 & $2.006 *$ & -0.09 & -1.587 & 0.057 & 0.661 & 0.25 & $2.463 *$ & 0.098 & 3.013* & -0.017 & -0.366 & 0.013 & 1.182 & 0.007 & 0.15 & 0.099 & $3.005 *$ & -0.027 & -0.598 & 0.013 & 1.182 & 0.014 & 0.283 \\
\hline 6 & 0.111 & $2.717 *$ & -0.11 & -1.537 & 0.154 & $2.529 *$ & 0.238 & $2.221 *$ & 0.119 & $2.988 *$ & -0.027 & \begin{tabular}{|c|}
-0.462 \\
\end{tabular} & 0.01 & 1.033 & $-2 \mathrm{E}-04$ & -0.003 & 0.12 & $3.047 *$ & -0.038 & -0.65 & 0.01 & 1.033 & 0.007 & 0.133 \\
\hline 7 & 0.137 & $2.449 *$ & -0.107 & -1.318 & & & & & 0.139 & 2.413* & -0.012 & -0.195 & 0.012 & 1.023 & -0.005 & -0.095 & 139 & $2.505 *$ & -0.02 & -0.31 & 12 & 1.023 & .008 & 0.142 \\
\hline 8 & $\begin{array}{l}0.104 \\
\end{array}$ & 1.47 & 0.026 & 0.378 & & & & & 0.12 & 1.804 & 0.074 & 1.08 & 3E-04 & 0.026 & -0.009 & -0.156 & $\begin{array}{l}0.112 \\
\end{array}$ & 1.747 & 0.093 & 1.368 & 3 & 0.194 & 0.008 & 0.143 \\
\hline 9 & 0.098 & 1.142 & -0.03 & -0.412 & & & & & |101| & 1.212 & 0.083 & 0.741 & -0.01 & -0.637 & -0.026 & -0.394 & 0.095 & 1.14 & 0.052 & 0.668 & -0.017 & -1.105 & -0.007 & -0.104 \\
\hline 10 & 0.106 & 0.974 & -0.06 & -0.522 & & & & & \begin{tabular}{|c|}
0.093 \\
\end{tabular} & 0.861 & 0.016 & \begin{tabular}{|c|c|}
0.113 \\
\end{tabular} & -0.04 & -1.236 & -0.045 & -0.611 & 0.106 & 1.053 & 0.07 & 0.652 & -0.05 & -1.459 & -0.032 & -0.421 \\
\hline
\end{tabular}

Non-Linear Granger Causality Test - FRANCE

\begin{tabular}{|c|c|c|c|c|c|c|c|c|c|c|c|c|c|c|c|c|c|c|c|c|c|c|c|c|}
\hline \multirow[b]{2}{*}{$\mathrm{Lx}=\mathrm{Ly}$} & \multicolumn{2}{|c|}{ E-GDP } & \multicolumn{2}{|c|}{ GDP-E } & \multicolumn{2}{|c|}{$\mathrm{C}-\mathrm{Y}$} & \multicolumn{2}{|c|}{$\mathrm{Y}-\mathrm{C}$} & \multicolumn{2}{|c|}{ E-GDP/N } & \multicolumn{2}{|c|}{ GDP/N-E } & \multicolumn{2}{|c|}{ E/GDP-GDP/N } & \multicolumn{2}{|c|}{ GDP/N-E/GDP } & \multicolumn{2}{|c|}{ E/N-GDP/N } & \multicolumn{2}{|c|}{ GDP/N-E/N } & \multicolumn{2}{|c|}{ E/GDP-GDP } & \multicolumn{2}{|c|}{ GDP-E/GDP } \\
\hline & Cs & \begin{tabular}{|c|} 
T-Val \\
\end{tabular} & Cs & $\mathrm{T}-\mathrm{V}$ & $\mathrm{Cs}$ & T- & $s$ & T- & Cs & T-Val & Cs & T-Val & Cs & $\mathrm{T}-\mathrm{Val}$ & Cs & \begin{tabular}{|c|} 
T-Val \\
\end{tabular} & Cs & T-Val & Cs & T-Val & Cs & $\mathrm{T}-\mathrm{Val}$ & Cs & T-Val \\
\hline 1 & -0.003 & \begin{tabular}{|l|}
-0.449 \\
\end{tabular} & .00 & & .032 & & 0.01 & & 0.002 & & -0.004 & & & & .003 & & E-04 & & 004 & & .009 & & 007 & 931 \\
\hline 2 & 1 & .829 & & 998 & & & 025 & & & & & & & & & & & & & & & & & 232 \\
\hline 3 & & & & -1.236 & & & & & & & & & & & & & & & & & & & & 371 \\
\hline 4 & & & & & & 473 & & & & & & & & & & & & & & & & & & 35 \\
\hline 5 & & & & & & 775 & & & & & & & & & & & & & & & & & & \\
\hline 6 & & & & & & 35 & & & & & & & & & & & & & & & & & & \\
\hline 7 & & & & & & & & & & & & & & & & & & & & & & .226 & & \\
\hline 8 & & & & -0.1 & & 1 & & & & & & & & & & .344 & & & & & & -0.3 & & 327 \\
\hline 9 & & & & 0.293 & & 1.093 & & & $0 \mathrm{JJ}$ & & & & & & & 0. & & & & & 2 & -0. & & 0.15 \\
\hline 10 & 0.061 & 367 & 0.004 & 0.082 & |.181| & 1.633 & .067 & 1.166 & 0.03 & \begin{tabular}{|l|}
1.097 \\
\end{tabular} & -0.003 & \begin{tabular}{|l|}
-0.073 \\
\end{tabular} & 0. & .04 & 0.012 & 311 & 0.0 & 0.794 & & 319 & \begin{tabular}{|l|}
-0.025 \\
\end{tabular} & -0.781 & 0.019 & .491 \\
\hline
\end{tabular}

Non-Linear Granger Causality Test - GERMANY

\begin{tabular}{|c|c|c|c|c|c|c|c|c|c|c|c|c|c|c|c|c|c|c|c|c|c|c|c|c|}
\hline \multirow[b]{2}{*}{$\mathrm{Lx}=\mathrm{Ly}$} & \multicolumn{2}{|c|}{ E-GDP } & \multicolumn{2}{|c|}{ GDP-E } & \multicolumn{2}{|c|}{$\overline{C-Y}$} & \multicolumn{2}{|c|}{$\overline{Y-C}$} & \multicolumn{2}{|c|}{ E-GDP/N } & \multicolumn{2}{|c|}{ GDP/N-E } & \multicolumn{2}{|c|}{ E/GDP-GDP/N } & \multicolumn{2}{|c|}{ GDP/N-E/GDP } & \multicolumn{2}{|c|}{ E/N-GDP/N } & \multicolumn{2}{|c|}{ GDP/N-E/N } & \multicolumn{2}{|c|}{ E/GDP-GDP } & \multicolumn{2}{|c|}{ GDP-E/GDP } \\
\hline & Cs & T-V & Cs & $\overline{T-V}$ & s & $\mathrm{T}$ - & Cs & $\overline{T-1}$ & Cs & T-Val & Cs & T-Val & Cs & \begin{tabular}{|c|} 
T-Val \\
\end{tabular} & Cs & T-Val & Cs & T-Val & Cs & T-Val & Cs & T-Val & Cs & T-Val \\
\hline 1 & 0.002 & 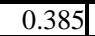 & $\overline{.00}$ & & & & & & & & & & & & & & \begin{tabular}{|c|}
$\mathrm{E}-04$ \\
\end{tabular} & 0.04 & & & 0.02 & & -04 & \\
\hline 2 & & & & & & & & & & & & & & & & & & & & & & & & \\
\hline 3 & & & & & & & & & & & & & & & & & & & & & & & & \\
\hline 4 & & & & & & & & & & & & & & & & & & & & & & & & \\
\hline 5 & 0.0 & & & -0.253 & & & & & & & & & & & & & & & & & & & & \\
\hline 6 & & & & 409 & & & & & & & & & & & & & & & & & & & & 25 \\
\hline 7 & & & & .097 & -0 & & & & & & & & & & & 28 & & & & & & & & 157 \\
\hline 8 & 0.1 & & 6 & -0.465 & -0 & -0.425 & -0.054 & -0.678 & & -0 & & -1. & & & & & 46 & 217 & & & 35 & 0.912 & 03 & -0.088 \\
\hline 9 & 0.072 & 53 & 1 & -0.791 & 0. & & & & & & & -1. & & 34 & & & 74 & 397 & & & & 54 & & 0.471 \\
\hline 10 & 0.085 & 1.167 & -0.079 & -1.198 & -0.061 & -0.333 & -0.15 & \begin{tabular}{|c|}
-0.753 \\
\end{tabular} & 0.026 & 0.595 & -0.095 & \begin{tabular}{|l|}
-1.229 \\
\end{tabular} & \begin{tabular}{c|}
0.059 \\
\end{tabular} & 1.217 & \begin{tabular}{|c|}
-0.09 \\
\end{tabular} & \begin{tabular}{|c|}
-1.217 \\
\end{tabular} & 0.064 & 0.685 & $\begin{array}{l}-0.095 \\
\end{array}$ & \begin{tabular}{|l|}
-1.229 \\
\end{tabular} & |0.085 & 1.253 & 0.026 & 0.59 \\
\hline
\end{tabular}


Non-Linear Granger Causality Test - GREECE

\begin{tabular}{|c|c|c|c|c|c|c|c|c|c|c|c|c|c|c|c|c|c|c|c|c|c|c|c|c|}
\hline \multirow[b]{2}{*}{$\mathrm{Lx}=\mathrm{Ly}$} & \multicolumn{2}{|c|}{ E-GDP } & \multicolumn{2}{|c|}{ GDP-E } & \multicolumn{2}{|c|}{ C-Y } & \multicolumn{2}{|c|}{$\mathrm{Y}-\mathrm{C}$} & \multicolumn{2}{|c|}{ E-GDP/N } & \multicolumn{2}{|c|}{ GDP/N-E } & \multicolumn{2}{|c|}{ E/GDP-GDP/N } & \multicolumn{2}{|c|}{ GDP/N-E/GDP } & \multicolumn{2}{|c|}{$\mathrm{E} / \mathrm{N}-\mathrm{GDP} / \mathrm{N}$} & \multicolumn{2}{|c|}{ GDP/N-E/N } & \multicolumn{2}{|c|}{ E/GDP-GDP } & \multicolumn{2}{|c|}{ GDP-E/GDP } \\
\hline & $\overline{\mathrm{Cs}}$ & T-Val & Cs & T-Val & $\mathrm{Cs}$ & T-Val & Cs & T-Val & $\mathrm{Cs}$ & $\mathrm{T}-\mathrm{Va}$ & $\overline{\mathrm{Cs}}$ & $\mathrm{T}-\mathrm{Va}$ & $\mathrm{Cs}$ & T-Val & $\overline{C s}$ & T-Val & Cs & $\mathrm{T}-\mathrm{Val}$ & Cs & $\mathrm{T}-\mathrm{Val}$ & $\mathrm{Cs}$ & $\mathrm{T}-\mathrm{Val}$ & $\mathrm{Cs}$ & T-Val \\
\hline 1 & 0.045 & 1.149 & 0.032 & 0.806 & 0.032 & 1.419 & -0.013 & .097 & 043 & 1.097 & 0.04 & 1.09 & 01 & -0.8 & .037 & 35 & 0.009 & 0.778 & 001 & -0.147 & .008 & -0.574 & 039 & .975 \\
\hline 2 & 0.054 & 1.122 & 014 & 0.348 & 023 & 0.733 & 009 & 0.268 & 7 & 0.989 & 03 & 0.808 & 07 & 35 & 22 & 55 & .007 & 296 & 11 & 72 & 01 & .048 & 022 & 537 \\
\hline 3 & 0.049 & 1.024 & 006 & 107 & 034 & 0.917 & -0.017 & -0.344 & 2 & 0.848 & 13 & .2 & 08 & 0.33 & 01 & 14 & -0.043 & -0.763 & 773 & 1.682 & & 0.939 & 06 & .112 \\
\hline 4 & 0.037 & 0.71 & 0.023 & -0.348 & 0.067 & 1.534 & -0.029 & -0.4 & 3 & 0.226 & 0.012 & -0.24 & -0.013 & 0.466 & 021 & 0.405 & 0 & & 054 & 1.649 & 3 & 0.552 & 13 & 225 \\
\hline 5 & 0.094 & 1.42 & -0.024 & -0.325 & 0.052 & 1.105 & $\mid-0.016$ & -0.2 & 2 & 1.304 & .028 & .4 & .018 & 0.291 & .01 & 0.14 & 0.071 & 1.089 & 102 & 1.767 & 0.035 & 0.581 & -0.015 & -0.18 \\
\hline 6 & 0.078 & 0.997 & -0.13 & $-2.313 *$ & 0.064 & 0.931 & 0.017 & 0.246 & .079 & 0.981 & -0.103 & $-1.997 *$ & -0.083 & -1.391 & 0.103 & 1.541 & 0.127 & 1.291 & .106 & 1.611 & -0.034 & -0.615 & -0.13 & -1.647 \\
\hline 7 & 0.212 & $2.689 *$ & 0.003 & 0.034 & 137 & 1.461 & 0.076 & 0.858 & 169 & $2.101^{*}$ & 031 & 0.451 & 0.096 & 1.43 & -0.034 & -0.419 & 0.055 & 0.818 & 109 & 1.445 & 0.105 & 1.776 & -0.061 & -0.677 \\
\hline 8 & 0.146 & 639* & 0.006 & 0.057 & 0.188 & $2.012^{*}$ & 68 & 1.431 & 146 & $3.434^{*}$ & 027 & 0.335 & 106 & 1.339 & -0.076 & $\begin{array}{c}-0.809 \\
\end{array}$ & 0.011 & 0.131 & 113 & 1.371 & 0.102 & 1.424 & $\begin{array}{l}-0.109 \\
\end{array}$ & -1.027 \\
\hline 9 & 0.144 & $2.366 *$ & 0.047 & 0.495 & 0.184 & 1.65 & 0.128 & 1.205 & 0.128 & $2.76^{*}$ & 0.077 & 1.035 & 0.113 & 1.153 & -0.05 & -0.487 & -0.071 & -0.61 & 0.092 & 0.915 & 0.096 & 1.099 & -0.039 & -0.33 \\
\hline 10 & 0.121 & 1.868 & 0.068 & 0.651 & 0.233 & $2.12 *$ & 0.017 & 0.135 & 0.1 & $2.287^{*}$ & 0.094 & 1.136 & 0.055 & 0.616 & 0.026 & 0.333 & -0.227 & -1.373 & 0.259 & 1.951 & 0.055 & 0.616 & 0.046 & 0.476 \\
\hline
\end{tabular}

\section{Non-Linear Granger Causality Test - IRELAND}

\begin{tabular}{|c|c|c|c|c|c|c|c|c|c|c|c|c|c|c|c|c|c|c|c|c|c|c|c|c|}
\hline \multirow[b]{2}{*}{$\mathrm{Lx}=\mathrm{Ly}$} & \multicolumn{2}{|c|}{ E-GDP } & \multicolumn{2}{|c|}{ GDP-E } & \multicolumn{2}{|c|}{$\mathrm{C}-\mathrm{Y}$} & \multicolumn{2}{|c|}{$\mathrm{Y}-\mathrm{C}$} & \multicolumn{2}{|c|}{ E-GDP/N } & \multicolumn{2}{|c|}{ GDP/N-E } & \multicolumn{2}{|c|}{ E/GDP-GDP/N } & \multicolumn{2}{|c|}{ GDP/N-E/GDP } & \multicolumn{2}{|c|}{ E/N-GDP/N } & \multicolumn{2}{|c|}{ GDP/N-E/N } & \multicolumn{2}{|c|}{ E/GDP-GDP } & \multicolumn{2}{|c|}{ GDP-E/GDP } \\
\hline & Cs & T-Val & Cs & T-Val & Cs & T-Val & Cs & T-Val & Cs & T-Val & Cs & T-Val & Cs & T-Val & Cs & T-Val & Cs & T-Val & Cs & T-Val & Cs & T-Val & Cs & T-Val \\
\hline 1 & 0.005 & 0.222 & 0.008 & 0.445 & \begin{tabular}{|c|}
0.039 \\
\end{tabular} & 1.051 & \begin{tabular}{|c|}
0.009 \\
\end{tabular} & 0.758 & -0.032 & $-1.972 *$ & -0.012 & \begin{tabular}{|c|}
-0.647 \\
\end{tabular} & 0.038 & 1.09 & 0.016 & 0.933 & \begin{tabular}{|c|}
-0.013 \\
\end{tabular} & -0.985 & 0.007 & 0.45 & \begin{tabular}{|c|}
-0.008 \\
\end{tabular} & 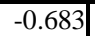 & 0.014 & 1.005 \\
\hline 2 & 0 & $\begin{array}{c}-0.001 \\
\end{array}$ & 0.006 & 0.24 & 0.04 & 0.91 & 0.032 & 1.55 & -0.065 & $-1.981 *$ & 0.011 & 0.314 & 0.062 & 1.241 & 0.003 & 0.112 & -0.011 & -0.467 & 0.005 & 0.133 & \begin{tabular}{|c|}
-0.014 \\
\end{tabular} & -0.564 & 0.012 & 556 \\
\hline 3 & \begin{tabular}{|l|l|}
0.019 \\
\end{tabular} & 0.475 & 0.015 & 0.341 & 0.013 & 0.24 & 0.017 & 0.413 & -0.07 & -1.716 & 0.015 & 0.326 & 0.06 & 1.182 & -0.007 & -0.238 & $-1 \mathrm{E}-04$ & -0.003 & -0.007 & -0.149 & 0.024 & -0.951 & 0.013 & 0.426 \\
\hline 4 & -0.014 & -0.269 & 0.042 & 0.65 & -0.045 & \begin{tabular}{|l|}
-0.583 \\
\end{tabular} & 0.067 & 1.957 & -0.068 & -1.572 & 0.024 & 0.417 & 0.083 & 1.304 & 0.011 & 0.332 & 0.018 & 0.519 & -0.029 & -0.472 & -0.065 & -1.412 & -0.009 & -0.26 \\
\hline 5 & 0.007 & 0.136 & 0.1 & 1.502 & -0.042 & \begin{tabular}{|l|l|}
-0.454 \\
\end{tabular} & 0.14 & 1.467 & -0.064 & -1.481 & 0.039 & 0.842 & 43 & 0.984 & 0.04 & 1.087 & 0.015 & 0.388 & 0.04 & 0.992 & -0.024 & -0.57 & -0.042 & -1.226 \\
\hline 6 & \begin{tabular}{|l|}
-0.052 \\
\end{tabular} & -0.742 & 0.111 & 1.105 & -0.18 & -0.806 & 0.131 & 0.894 & -0.095 & -1.487 & -0.013 & \begin{tabular}{|l|}
-0.264 \\
\end{tabular} & 0.042 & 1.319 & 0.015 & 0.385 & 0.039 & 0.93 & 0.01 & 0.184 & -0.04 & -0.625 & -0.011 & -0.224 \\
\hline 7 & -0.184 & -1.63 & 0.048 & 0.366 & 0.021 & 0.094 & 0.016 & 0.078 & -0.104 & -1.232 & -0.011 & -0.221 & 0.034 & 0.913 & 0.01 & 0.29 & 0.033 & 0.785 & 0.032 & 0.598 & 0.015 & 0.225 & 0.037 & 0.458 \\
\hline 8 & $\mid-0.109$ & -0.593 & 0.346 & $2.639 *$ & & & & & \begin{tabular}{|l|l|}
-0.124 \\
\end{tabular} & -1.022 & $\begin{array}{l}-0.09 \\
\end{array}$ & \begin{tabular}{|c|}
-0.907 \\
\end{tabular} & 0.053 & 1.244 & 0.018 & 0.336 & 0.021 & 0.48 & 0.036 & 0.636 & -0.025 & -0.271 & 0.066 & 0.869 \\
\hline 9 & -0.233 & -0.787 & 0.294 & $1.978 *$ & & & & & \begin{tabular}{|l}
-0.158 \\
\end{tabular} & -0.879 & 0.003 & 0.015 & 0.066 & 1.123 & 0.0 & 0.739 & -0.0 & -0.103 & 0.096 & 1.591 & 0.077 & 0.538 & 0.034 & 0.221 \\
\hline 10 & & & 0.307 & 1.828 & & & & & -0.285 & -1.315 & 0.126 & $2.786 *$ & 0.047 & 0.634 & 0.008 & 0.125 & 0.019 & 0.323 & 0.068 & 0.76 & -0.025 & -0.114 & 0 & \\
\hline
\end{tabular}

Non-Linear Granger Causality Test - ITALY

\begin{tabular}{|c|c|c|c|c|c|c|c|c|c|c|c|c|c|c|c|c|c|c|c|c|c|c|c|c|}
\hline \multirow[b]{2}{*}{$\mathrm{Lx}=\mathrm{Ly}$} & \multicolumn{2}{|c|}{$\overline{\text { E-GDP }}$} & \multicolumn{2}{|c|}{ GDP-E } & \multicolumn{2}{|c|}{$\mathrm{C}-\mathrm{Y}$} & \multicolumn{2}{|c|}{$\mathrm{Y}-\mathrm{C}$} & \multicolumn{2}{|c|}{ E-GDP/N } & \multicolumn{2}{|c|}{ GDP/N-E } & \multicolumn{2}{|c|}{ E/GDP-GDP/N } & \multicolumn{2}{|c|}{ GDP/N-E/GDP } & \multicolumn{2}{|c|}{ E/N-GDP/N } & \multicolumn{2}{|c|}{ GDP/N-E/N } & \multicolumn{2}{|c|}{ E/GDP-GDP } & \multicolumn{2}{|c|}{ GDP-E/GDP } \\
\hline & Cs & T-Val & Cs & T-Val & Cs & T-V & $\mathrm{Cs}$ & T-I & Cs & T-Val & Cs & $\mathrm{T}-\mathrm{Val}$ & Cs & $\mathrm{T}$-Val & Cs & -Val & Cs & $\mathrm{T}-\mathrm{Val}$ & Cs & T-Val & Cs & T-Val & Cs & -Val \\
\hline 1 & -0.019 & 0.811 & -0.044 & \begin{tabular}{|l|l}
-1.898 \\
\end{tabular} & 0.015 & 0.593 & 0.03 & 1.309 & -0.022 & & -0.036 & 1.62 & & & -0.019 & & -0.036 & \begin{tabular}{|l|}
-1.62 \\
\end{tabular} & -0.036 & \begin{tabular}{|l|}
-1.62 \\
\end{tabular} & -0.006 & -0.322 & -0.023 & -1.375 \\
\hline 2 & 0.0 & 55 & 5E-04 & 0.014 & 8 & & .072 & & .05 & & & & & 59 & & & & 44 & & & & & & 56 \\
\hline 3 & & & & I & & & & & 0. & & & & & & & & & & & & & & & \\
\hline 4 & 0.0 & tit & & 1.6 & & & & & & & & & & 56 & & & & & & & & & & \\
\hline 5 & & & & 1.393 & 0 & 0. & & & 51 & & 67 & 22 & & 88 & & & & 22 & & & & & & 96 \\
\hline 6 & & & 2 & 1.283 & -0 & & & & 46 & & & & & & & & & & & & & & & 44 \\
\hline 7 & & 37 & & 1.173 & & & & 1.1 & & & & & & & & & & & & & & & & .629 \\
\hline 8 & & & & 0 & & & & & & & & & & & & & & & & & & & & - \\
\hline 9 & $\begin{array}{c}-0.056 \\
\end{array}$ & .577 & 0 & .534 & -0.0 & & & & & & 17 & $1.997^{*}$ & & & 0.0 & & & & & & & & & .01 \\
\hline 10 & 0.167 & 1.17 & 0.003 & 0.024 & -0.03 & -0.538 & 0.037 & 0.566 & & & .191 & $2.101 *$ & & & 2 & $2.029 *$ & 0.191 & $2.101 *$ & 0.191 & $2.101 *$ & 0.064 & 1.204 & 0.027 & 0.322 \\
\hline
\end{tabular}


Non-Linear Granger Causality Test - LUXEMBOURG

\begin{tabular}{|c|c|c|c|c|c|c|c|c|c|c|c|c|c|c|c|c|c|c|c|c|c|c|c|c|}
\hline \multirow[b]{2}{*}{$\mathrm{Lx}=\mathrm{Ly}$} & \multicolumn{2}{|c|}{ E-GDP } & \multicolumn{2}{|c|}{ GDP-E } & \multicolumn{2}{|c|}{$\mathrm{C}-\mathrm{Y}$} & \multicolumn{2}{|c|}{$\mathrm{Y}-\mathrm{C}$} & \multicolumn{2}{|c|}{ E-GDP/N } & \multicolumn{2}{|c|}{ GDP/N-E } & \multicolumn{2}{|c|}{ E/GDP-GDP/N } & \multicolumn{2}{|c|}{ GDP/N-E/GDP } & \multicolumn{2}{|c|}{ E/N-GDP/N } & \multicolumn{2}{|c|}{ GDP/N-E/N } & \multicolumn{2}{|c|}{ E/GDP-GDP } & \multicolumn{2}{|c|}{ GDP-E/GDP } \\
\hline & $\mathrm{Cs}$ & T-Val & Cs & T-Val & Cs & T-Val & Cs & T-Val & Cs & $\mathrm{T}-\mathrm{Val}$ & $\mathrm{Cs}$ & T-Val & $\mathrm{Cs}$ & T-Val & Cs & T-Val & Cs & T-Val & $\mathrm{Cs}$ & T-Val & $\mathrm{Cs}$ & T-Val & $\mathrm{Cs}$ & T-Val \\
\hline 1 & $\begin{array}{c}-0.007 \\
\end{array}$ & -0.46 & 0.004 & 0.318 & \begin{tabular}{|l|}
-0.024 \\
\end{tabular} & -1.205 & \begin{tabular}{|l|}
-0.015 \\
\end{tabular} & -1.424 & -0.014 & -0.992 & \begin{tabular}{|c|}
0.006 \\
\end{tabular} & 0.444 & 0.003 & 0.396 & 0.014 & 1.078 & 6E-04 & 0.072 & \begin{tabular}{|c|}
0.003 \\
\end{tabular} & \begin{tabular}{|c|}
0.241 \\
\end{tabular} & \begin{tabular}{|c|}
-0.003 \\
\end{tabular} & \begin{tabular}{|l|}
-0.961 \\
\end{tabular} & |0.015 & 1.251 \\
\hline 2 & -0.038 & -1.332 & $\begin{array}{c}-0.036 \\
\end{array}$ & -1.471 & \begin{tabular}{|l|l|}
0.046 \\
\end{tabular} & 1.746 & \begin{tabular}{|c|}
-0.043 \\
\end{tabular} & -1.493 & \begin{tabular}{|c|}
-0.044 \\
\end{tabular} & -1.702 & -0.039 & -1.214 & 0.002 & 0.233 & 0.009 & 0.478 & \begin{tabular}{|c|}
-0.036 \\
\end{tabular} & \begin{tabular}{|c|}
-1.43 \\
\end{tabular} & \begin{tabular}{|c|}
-0.044 \\
\end{tabular} & \begin{tabular}{|l|}
-1.265 \\
\end{tabular} & \begin{tabular}{|c|}
-0.005 \\
\end{tabular} & \begin{tabular}{|c|}
-0.976 \\
\end{tabular} & 0.017 & 1.158 \\
\hline 3 & -0.078 & -1.442 & 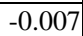 & -0.215 & 0.235 & 1.75 & \begin{tabular}{|c|}
-0.068 \\
\end{tabular} & -1.718 & \begin{tabular}{|c|}
-0.089 \\
\end{tabular} & -1.902 & \begin{tabular}{|c|}
0.007 \\
\end{tabular} & 0.224 & 0.004 & 0.222 & 0.005 & \begin{tabular}{|c|}
0.181 \\
\end{tabular} & \begin{tabular}{|c|}
-0.083 \\
\end{tabular} & \begin{tabular}{|l|}
-1.645 \\
\end{tabular} & \begin{tabular}{|c|}
-0.008 \\
\end{tabular} & \begin{tabular}{|l|}
-0.176 \\
\end{tabular} & \begin{tabular}{|c|}
-0.006 \\
\end{tabular} & \begin{tabular}{|l|}
-0.993 \\
\end{tabular} & |008 & 0.321 \\
\hline 4 & -0.087 & -1.459 & -0.099 & -1.858 & 0.231 & 1.604 & 0 & -999 & -0.087 & $-2.007 *$ & -0.099 & -1.608 & 0.013 & 0.763 & -0.032 & -1.235 & -0.08 & -1.648 & -0.04 & -0.644 & -0.01 & -1.043 & -0.013 & -0.52 \\
\hline 5 & $\begin{array}{l}-0.008 \\
\end{array}$ & $\begin{array}{c}-0.109 \\
\end{array}$ & \begin{tabular}{|c|}
-0.073 \\
\end{tabular} & -1.025 & 0.111 & 1.014 & & & \begin{tabular}{|c|}
-0.099 \\
\end{tabular} & -1.292 & -0.073 & -0.751 & 0.045 & 1.933 & -0.014 & \begin{tabular}{|c|}
-0.315 \\
\end{tabular} & -0.039 & \begin{tabular}{|c|}
-0.598 \\
\end{tabular} & \begin{tabular}{|c|}
-0.073 \\
\end{tabular} & \begin{tabular}{|c|}
-0.68 \\
\end{tabular} & 0 & \begin{tabular}{|c|}
-999 \\
\end{tabular} & \begin{tabular}{|c|}
0.013 \\
\end{tabular} & 0.348 \\
\hline 6 & $\begin{array}{l}-0.028 \\
\end{array}$ & -0.249 & -0.169 & -1.374 & & & & & \begin{tabular}{|c|c|}
-0.153 \\
\end{tabular} & -1.154 & & & 0.071 & 1.917 & -0.016 & -0.371 & -0.074 & -0.725 & -0.044 & -0.57 & 0 & \begin{tabular}{|c|}
-999 \\
\end{tabular} & 0.024 & 0.439 \\
\hline 7 & 0.143 & 1.458 & -0.286 & $-2.442 *$ & & & & & $\begin{array}{l}0.143 \\
\end{array}$ & 1.458 & & & 0.107 & 1.646 & -0.079 & \begin{tabular}{|l|}
-1.186 \\
\end{tabular} & \begin{tabular}{|c|}
0.077 \\
\end{tabular} & 0.8 & \begin{tabular}{|c|}
-0.193 \\
\end{tabular} & \begin{tabular}{|l|}
-1.829 \\
\end{tabular} & 0 & 0 & -0.036 & \begin{tabular}{|l|l|}
-0.399 \\
\end{tabular} \\
\hline 8 & 0.167 & 1.308 & & & & & & & 0.167 & 1.308 & & & 0.036 & 0.276 & -0.076 & \begin{tabular}{|l|}
-0.397 \\
\end{tabular} & 0.083 & 0.775 & & & -0.114 & \begin{tabular}{|l|}
-1.317 \\
\end{tabular} & -0.183 & -1.062 \\
\hline 9 & & & & & & & & & & & & & 0.5 & 1.85 & 0.2 & $2.259 *$ & & & & & 0.5 & 1.85 & 0.2 & $2.259^{*}$ \\
\hline 10 & & & & & & & & & & & & & & & & & & & & & & & & \\
\hline
\end{tabular}

Non-Linear Granger Causality Test - NETHERLANDS

\begin{tabular}{|c|c|c|c|c|c|c|c|c|c|c|c|c|c|c|c|c|c|c|c|c|c|c|c|c|}
\hline \multirow[b]{2}{*}{$\mathrm{Lx}=\mathrm{Ly}$} & \multicolumn{2}{|c|}{ E-GDP } & \multicolumn{2}{|c|}{ GDP-E } & \multicolumn{2}{|c|}{$\mathrm{C}-\mathrm{Y}$} & \multicolumn{2}{|c|}{ Y-C } & \multicolumn{2}{|c|}{ E-GDP/N } & \multicolumn{2}{|c|}{ GDP/N-E } & \multicolumn{2}{|c|}{ E/GDP-GDP/N } & \multicolumn{2}{|c|}{ GDP/N-E/GDP } & \multicolumn{2}{|c|}{ E/N-GDP/N } & \multicolumn{2}{|c|}{ GDP/N-E/N } & \multicolumn{2}{|c|}{ E/GDP-GDP } & \multicolumn{2}{|c|}{ GDP-E/GDP } \\
\hline & $\mathrm{Cs}$ & T-Val & $\mathrm{Cs}$ & T-Val & $\mathrm{Cs}$ & T-Val & $\mathrm{Cs}$ & T-Val & $\mathrm{Cs}$ & T-Val & $\mathrm{Cs}$ & T-Val & $\mathrm{Cs}$ & T-Val & $\mathrm{Cs}$ & T-Val & $\mathrm{Cs}$ & T-Val & $\mathrm{Cs}$ & T-Val & $\mathrm{Cs}$ & T-Val & $\mathrm{Cs}$ & T-Val \\
\hline 1 & $\mid-0.013$ & -1.109 & -0.004 & $\begin{array}{l}-0.249 \\
\end{array}$ & 0.006 & 0.229 & 0.024 & 0.649 & 0.009 & 0.735 & -0.003 & -0.149 & -0.023 & -1.852 & 0.005 & 0.259 & -0.005 & -0.43 & -0.003 & -0.154 & -0.024 & $-2.149 *$ & 0.007 & 0.422 \\
\hline 2 & -0.016 & -1.036 & -0.012 & -0.515 & -0.019 & -0.404 & 0.019 & 0.418 & 0.018 & 1.094 & -0.005 & -0.222 & -0.035 & -1.834 & 0.007 & 0.365 & -0.003 & -0.212 & -0.008 & -0.383 & -0.039 & $-2.036^{*}$ & 0.002 & .095 \\
\hline 3 & \begin{tabular}{|c|}
-0.033 \\
\end{tabular} & -1.157 & \begin{tabular}{|c|c|}
-0.037 \\
\end{tabular} & -1.134 & -0.026 & -0.465 & 0.035 & 0.698 & 0.014 & 0.588 & -0.01 & -0.4 & -0.068 & $-2.188 *$ & -0.007 & -0.308 & -0.016 & -0.68 & -0.026 & -0.844 & -0.066 & $-2.064 *$ & -0.016 & -0.613 \\
\hline 4 & $\mid-0.069$ & -1.495 & -0.062 & -1.388 & -0.086 & -1.092 & 0.018 & 0.234 & -0.026 & -0.724 & -0.021 & 0.646 & -0.099 & $-2.331 *$ & -0.02 & -0.685 & -0.074 & -1.765 & -0.049 & -1.299 & -0.088 & $-2.127 *$ & -0.037 & -1.152 \\
\hline 5 & $\mid-0.098$ & -1.333 & -0.112 & -1.804 & -0.261 & -1.932 & 0.162 & 1.801 & -0.019 & -0.397 & -0.032 & -0.717 & -0.134 & $-2.189 *$ & -0.088 & -1.924 & -0.09 & -1.389 & -0.075 & -1.465 & -0.12 & $-2.027 *$ & -0.114 & $-2.258 *$ \\
\hline 6 & $\mid-0.239$ & -1.889 & -0.058 & -0.893 & -0.384 & $-1.987^{*}$ & 0.152 & 1.658 & -0.059 & -0.775 & 0.026 & 0.579 & -0.219 & $-2.265^{*}$ & -0.044 & -0.575 & -0.199 & -1.702 & 0.009 & 0.136 & -0.247 & $-2.284 *$ & -0.099 & -1.487 \\
\hline 7 & $\mid-0.238$ & -1.488 & \begin{tabular}{|c|c|}
-0.127 \\
\end{tabular} & \begin{tabular}{|c|}
-011 \\
\end{tabular} & & & \begin{tabular}{|c|}
0.167 \\
\end{tabular} & 1.73 & -0.046 & -0.583 & 0.056 & \begin{tabular}{|c|}
1.184 \\
\end{tabular} & -0.156 & -1.006 & -0.003 & -0.029 & -0.179 & -1.328 & 0.051 & 0.705 & -0.21 & -1.177 & -0.14 & -1.159 \\
\hline 8 & $\mid-0.405$ & -1.363 & -0.046 & $\begin{array}{c}-0.322 \\
\end{array}$ & & & & & -0.15 & -1.364 & 0.034 & $\mid$\begin{tabular}{|l|l|}
0.511 \\
\end{tabular} & -0.288 & -1.801 & -0.069 & -0.508 & -0.397 & $-2.167 *$ & 0.006 & 0.05 & -0.304 & \begin{tabular}{|c|}
-1.48 \\
\end{tabular} & -0.096 & -0.702 \\
\hline 9 & & & & & & & & & -0.186 & -1.321 & -0.041 & -0.399 & & & -0.271 & -1.19 & & & & & & & & \\
\hline 10 & & & & & & & & & -0.207 & -1.072 & -0.202 & -1.167 & & & & & & & & & & & & \\
\hline
\end{tabular}

Non-Linear Granger Causality Test - PORTUGAL

\begin{tabular}{|c|c|c|c|c|c|c|c|c|c|c|c|c|c|c|c|c|c|c|c|c|c|c|c|c|}
\hline \multirow[b]{2}{*}{$\mathrm{Lx}=\mathrm{Ly}$} & \multicolumn{2}{|c|}{ E-GDP } & \multicolumn{2}{|c|}{ GDP-E } & \multicolumn{2}{|c|}{ C-Y } & \multicolumn{2}{|c|}{ Y-C } & \multicolumn{2}{|c|}{ E-GDP/N } & \multicolumn{2}{|c|}{ GDP/N-E } & \multicolumn{2}{|c|}{ E/GDP-GDP/N } & \multicolumn{2}{|c|}{ GDP/N-E/GDP } & \multicolumn{2}{|c|}{ E/N-GDP/N } & \multicolumn{2}{|c|}{ GDP/N-E/N } & \multicolumn{2}{|c|}{ E/GDP-GDP } & \multicolumn{2}{|c|}{ GDP-E/GDP } \\
\hline & $\mathrm{Cs}$ & T-Val & Cs & T-Val & $\mathrm{Cs}$ & T-Val & Cs & T-Val & Cs & T-Val & $\mathrm{Cs}$ & T-Val & $\overline{\mathrm{Cs}}$ & T-Val & $\mathrm{Cs}$ & T-Val & $\mathrm{Cs}$ & T-Val & $\mathrm{Cs}$ & T-Val & $\mathrm{Cs}$ & T-Val & Cs & T-Val \\
\hline 1 & 0.078 & 1.329 & 0.008 & 0.695 & 0.009 & 0.359 & 0.028 & 1.831 & 0.064 & 1.321 & 0.005 & 0.517 & -0.011 & -0.982 & 0.011 & 0.785 & 0.071 & 1.425 & 0.027 & 1.163 & -0.013 & -0.944 & 0.011 & 0.761 \\
\hline 2 & 0.058 & 1.077 & 0.01 & 0.685 & 0.015 & 0.362 & 0.061 & $2.014 *$ & 0.058 & 1.095 & 0.006 & 0.436 & -0.005 & -0.425 & 0.014 & 0.776 & 0.059 & 1.072 & 0.021 & 0.878 & -0.006 & -0.52 & 0.014 & 0.779 \\
\hline 3 & 0.054 & 0.878 & 0.012 & 0.64 & -0.001 & -0.033 & 0.067 & 1.625 & 0.055 & 0.909 & 0.006 & 0.314 & -0.015 & -0.718 & 0.018 & 0.775 & 0.054 & 0.841 & 0.025 & 0.771 & -0.017 & -0.761 & 0.018 & 0.78 \\
\hline 4 & 0.038 & 0.636 & 0.013 & 0.569 & 0.019 & 0.415 & 0.054 & 0.737 & 0.04 & 0.683 & 0.004 & 0.162 & 0.005 & 0.328 & 0.021 & 0.782 & 0.036 & 0.573 & 0.028 & 0.708 & 0.003 & 0.179 & 0.022 & 0.786 \\
\hline 5 & 0.023 & 0.33 & 0.015 & 0.496 & 0.057 & 0.599 & -0.003 & -0.028 & 0.028 & 0.394 & 0.042 & 1.001 & -0.016 & -0.596 & 0.027 & 0.805 & -0.002 & -0.047 & 0.074 & 1.165 & -0.02 & -0.63 & 0.03 & 0.782 \\
\hline 6 & 0.058 & 0.726 & 0.036 & 0.759 & 0.14 & $2.031^{*}$ & 0.065 & 0.326 & 0.063 & 0.784 & 0.036 & 0.771 & -0.04 & -0.831 & 0.05 & 0.873 & 0.009 & 0.118 & 0.096 & 1.21 & -0.045 & -0.885 & 0.052 & 0.881 \\
\hline 7 & 0.052 & 0.364 & -0.064 & -0.929 & 0.2 & $1.998^{*}$ & 0.269 & $2.516^{*}$ & 0.194 & $2.043^{\prime}$ & -0.028 & -0.396 & -0.092 & -0.964 & 0.025 & 0.359 & 0.185 & 1.575 & 0.067 & 0.682 & -0.092 & -1.108 & -0.011 & -0.178 \\
\hline 8 & & & & & & & & & & & & & & & & & & & & & & & 0.095 & 0.687 \\
\hline 9 & & & & & & & & & & & & & & & & & & & & & & & & \\
\hline 10 & & & & & & & & & & & & & & & & & & & & & & & & \\
\hline
\end{tabular}


Non-Linear Granger Causality Test - SPAIN

\begin{tabular}{|c|c|c|c|c|c|c|c|c|c|c|c|c|c|c|c|c|c|c|c|c|c|c|c|c|}
\hline \multirow[b]{2}{*}{$\mathrm{Lx}=\mathrm{Ly}$} & \multicolumn{2}{|c|}{ E-GDP } & \multicolumn{2}{|c|}{ GDP-E } & \multicolumn{2}{|c|}{$\mathrm{C}-\mathrm{Y}$} & \multicolumn{2}{|c|}{$\mathrm{Y}-\mathrm{C}$} & \multicolumn{2}{|c|}{ E-GDP/N } & \multicolumn{2}{|c|}{ GDP/N-E } & \multicolumn{2}{|c|}{ E/GDP-GDP/N } & \multicolumn{2}{|c|}{ GDP/N-E/GDP } & \multicolumn{2}{|c|}{ E/N-GDP/N } & \multicolumn{2}{|c|}{ GDP/N-E/N } & \multicolumn{2}{|c|}{ E/GDP-GDP } & \multicolumn{2}{|c|}{ GDP-E/GDP } \\
\hline & Cs & T-Val & Cs & T-Val & Cs & T-Val & Cs & T-Val & Cs & T-Val & Cs & T-Val & Cs & T-Val & Cs & T-Val & Cs & T-Val & Cs & $\mathrm{T}-\mathrm{Val}$ & Cs & T-Val & Cs & T-Val \\
\hline 1 & -0.004 & -0.388 & 0.004 & 0.457 & 0.006 & 0.614 & 0.037 & 2.88 * & 4E-04 & 0.038 & 0.005 & 0.303 & -0.013 & -0.592 & -0.004 & \begin{tabular}{|l|}
-0.159 \\
\end{tabular} & -0.002 & -0.166 & 0.009 & 1.036 & 0.01 & 0.381 & 0.013 & 0.562 \\
\hline 2 & -0.006 & -0.317 & -0.016 & -0.748 & 0.023 & 1.223 & 0.037 & $2.782 *$ & 0.003 & 0.167 & -0.026 & -0.848 & -0.014 & -0.309 & 0.029 & 0.846 & 0.001 & 0.078 & -0.019 & -0.949 & -0.053 & -1.295 & 0.061 & 1.928 \\
\hline 3 & 0.029 & 1.015 & -0.049 & -1.315 & 0.003 & 0.167 & 0.032 & 1.755 & 0.026 & 1.115 & -0.065 & -1.334 & -0.018 & -0.274 & 0.125 & $2.484 *$ & 0.057 & 1.687 & -0.036 & -1.138 & -0.048 & -0.738 & 0.053 & 1.303 \\
\hline 4 & -0.007 & -0.18 & -0.09 & -1.376 & 0.03 & 1.006 & 0.039 & 1.571 & -0.013 & -0.36 & \begin{tabular}{|l|l|}
-0.109 \\
\end{tabular} & -1.454 & -0.017 & -0.2 & 0.162 & $2.514 *$ & 0.011 & 0.306 & -0.083 & -1.354 & -0.137 & -1.622 & 0.056 & 0.715 \\
\hline 5 & 0.086 & 0.743 & -0.085 & -0.831 & 0.018 & 0.76 & 0.02 & 0.855 & 0.011 & 0.15 & -0.093 & -1.098 & -0.06 & -0.508 & 0.169 & $2.319 *$ & -0.008 & -0.113 & -0.099 & -1.159 & -0.222 & -1.329 & 0.007 & 0.073 \\
\hline 6 & -0.008 & -0.053 & -0.083 & -0.576 & -0.011 & -0.664 & -0.019 & -1.094 & -0.181 & -1.35 & -0.143 & -1.388 & -0.115 & -0.899 & 0.145 & $2.267 *$ & -0.167 & -1.571 & -0.138 & -1.773 & -0.152 & -0.814 & -0.161 & -0.911 \\
\hline 7 & 0.273 & 1.919 & 0.01 & 0.729 & -0.023 & -0.817 & -0.032 & -1.133 & 0.051 & 0.191 & 0.014 & 0.692 & & & 0.137 & 1.948 & -0.136 & -0.581 & -0.239 & -1.158 & & & -0.399 & -1.226 \\
\hline 8 & 0.286 & 1.438 & 0.012 & 0.74 & -0.042 & -0.938 & -0.057 & -1.241 & & & & & & & & & & & & & & & & \\
\hline 9 & & & & & -0.093 & -0.948 & -0.136 & -1.846 & & & & & & & & & & & & & & & & \\
\hline 10 & & & & & -0.11 & -0.638 & -0.4 & $-2.875 *$ & & & & & & & & & & & & & & & & \\
\hline
\end{tabular}

\begin{tabular}{|c|c|c|c|c|c|c|c|c|c|c|c|c|c|c|c|c|c|c|c|c|c|c|c|c|}
\hline \multirow[b]{2}{*}{$\mathrm{Lx}=\mathrm{Ly}$} & \multicolumn{2}{|c|}{ E-GDP } & \multicolumn{2}{|c|}{ GDP-E } & \multicolumn{2}{|c|}{$\mathrm{C}-\mathrm{Y}$} & \multicolumn{2}{|c|}{$\mathrm{Y}-\mathrm{C}$} & \multicolumn{2}{|c|}{ E-GDP/N } & \multicolumn{2}{|c|}{ GDP/N-E } & \multicolumn{2}{|c|}{ E/GDP-GDP/N } & \multicolumn{2}{|c|}{ GDP/N-E/GDP } & \multicolumn{2}{|c|}{$\mathrm{E} / \mathrm{N}-\mathrm{GDP} / \mathrm{N}$} & \multicolumn{2}{|c|}{ GDP/N-E/N } & \multicolumn{2}{|c|}{ E/GDP-GDP } & \multicolumn{2}{|c|}{ GDP-E/GDP } \\
\hline & $\mathrm{Cs}$ & T-Val & Cs & T-Val & Cs & T-Val & $\mathrm{Cs}$ & T-Val & $\mathrm{Cs}$ & T-Val & $\mathrm{Cs}$ & T-Val & Cs & $\mathrm{T}-\mathrm{Val}$ & $\mathrm{Cs}$ & T-Val & $\mathrm{Cs}$ & T-Val & $\mathrm{Cs}$ & T-Val & $\mathrm{Cs}$ & $\mathrm{T}-\mathrm{Val}$ & $\mathrm{Cs}$ & T-Val \\
\hline 1 & -0.012 & -0.594 & -0.016 & -1.143 & 0.004 & 0.146 & 0.029 & 0.636 & -0.012 & -0.476 & -0.017 & -1.149 & -0.004 & -0.266 & -0.011 & \begin{tabular}{|c|}
-1.276 \\
\end{tabular} & -0.014 & -0.581 & -0.014 & -1.004 & 0.054 & 1.644 & -0.031 & -1.726 \\
\hline 2 & 0.038 & \begin{tabular}{|l|l|}
0.799 \\
\end{tabular} & -0.015 & -0.474 & 0.003 & 0.119 & 0.034 & 0.747 & 0.058 & 1.191 & -0.013 & -0.414 & -0.017 & -0.714 & -0.023 & -1.09 & 0.061 & 1.262 & -0.014 & -0.47 & 0.065 & 1.68 & -0.068 & $-2.244^{*}$ \\
\hline 3 & 0.059 & \begin{tabular}{|l|l|}
0.846 \\
\end{tabular} & -0.079 & -1.114 & 0.025 & 0.485 & -0.042 & -0.682 & 0.061 & 0.976 & -0.044 & -0.783 & -0.025 & -0.617 & -0.017 & \begin{tabular}{|l|}
-0.529 \\
\end{tabular} & 0.048 & 0.751 & -0.054 & -0.96 & 0.047 & 0.709 & -0.097 & -1.704 \\
\hline 4 & 0.116 & 1.295 & $9 \mathrm{E}-04$ & 0.011 & 0.046 & 0.642 & 0.026 & 0.454 & 0.074 & 0.861 & 0.018 & 0.224 & -0.01 & -0.22 & 0.029 & 0.546 & 0.054 & 0.633 & 0.002 & 0.029 & 0.053 & 0.557 & -0.003 & -0.038 \\
\hline 5 & 0.133 & 1.213 & 0.054 & 0.644 & 0.057 & 0.558 & -0.068 & -0.577 & 0.106 & 0.887 & 0.046 & 0.528 & 0.023 & 0.512 & 0.07 & 1.274 & 0.033 & 0.271 & 0.052 & 0.614 & 0.041 & 0.271 & 0.011 & 0.096 \\
\hline 6 & 0.132 & 1.045 & $\overline{0.02}$ & 0.207 & 0.026 & 0.169 & -0.053 & -0.362 & 0.127 & 0.996 & 0.056 & \begin{tabular}{|l|}
0.517 \\
\end{tabular} & 0.078 & 1.624 & 0.069 & 1.062 & 0.068 & 0.586 & 0.053 & 0.513 & 0.082 & 0.584 & 0.018 & 0.14 \\
\hline 7 & 0.115 & 0.782 & 0.073 & 0.79 & & & & & 0.12 & 0.808 & 0.115 & 1.195 & 0.047 & 1.027 & 0.044 & 0.659 & 0.106 & 0.716 & 0.05 & 0.401 & 0.033 & 0.157 & -0.126 & -0.784 \\
\hline 8 & 0.025 & \begin{tabular}{|l|l|}
0.154 \\
\end{tabular} & 0.158 & 1.655 & & & & & 0.043 & 0.267 & 0.147 & 1.471 & 0.048 & 0.718 & 0.02 & 0.246 & 0.042 & 0.249 & 0.182 & 1.513 & -0.214 & -0.429 & & \\
\hline 9 & -0.083 & -0.44 & 0.206 & $2.16 *$ & & & & & -0.024 & -0.122 & 0.224 & 1.77 & 0.076 & 0.901 & 0.011 & 0.118 & 0.005 & 0.027 & 0.2 & 1.523 & & & & \\
\hline 10 & 0.028 & 0.131 & 0.225 & 1.464 & & & & & \begin{tabular}{|l|}
0.071 \\
\end{tabular} & 0.348 & 0.107 & \begin{tabular}{|l|l|} 
\\
\end{tabular} & 0.032 & 0.299 & 0.02 & 0.149 & 0.083 & 0.393 & 0.085 & 0.609 & & & & \\
\hline
\end{tabular}

Non-Linear Granger Causality Test - UK

\begin{tabular}{|c|c|c|c|c|c|c|c|c|c|c|c|c|c|c|c|c|c|c|c|c|c|c|c|c|}
\hline \multirow[b]{2}{*}{$\mathrm{Lx}=\mathrm{Ly}$} & \multicolumn{2}{|c|}{ E-GDP } & \multicolumn{2}{|c|}{ GDP-E } & \multicolumn{2}{|c|}{ C-Y } & \multicolumn{2}{|c|}{ Y-C } & \multicolumn{2}{|c|}{ E-GDP/N } & \multicolumn{2}{|c|}{ GDP/N-E } & \multicolumn{2}{|c|}{ E/GDP-GDP/N } & \multicolumn{2}{|c|}{ GDP/N-E/GDP } & \multicolumn{2}{|c|}{ E/N-GDP/N } & \multicolumn{2}{|c|}{ GDP/N-E/N } & \multicolumn{2}{|c|}{ E/GDP-GDP } & \multicolumn{2}{|c|}{ GDP-E/GDP } \\
\hline & $\mathrm{Cs}$ & T-Val & Cs & T-Val & $\mathrm{Cs}$ & T-Val & Cs & T-Val & Cs & T-Val & $\mathrm{Cs}$ & T-Val & Cs & T-Val & Cs & T-Val & $\mathrm{Cs}$ & T-Val & $\mathrm{Cs}$ & T-Val & & T-Val & Cs & T-Val \\
\hline 1 & -0.012 & -1.577 & -0.005 & -1.662 & 0.082 & 1.98 & 0.057 & 1.114 & -0.012 & -1.577 & -0.005 & -1.662 & 0 & -999 & 0 & -999 & -0.01 & -1.288 & -0.005 & -1.546 & 0 & -999 & 0 & -999 \\
\hline 2 & -0.024 & -1.524 & -0.011 & -1.784 & 0.109 & $2.277 *$ & 0.031 & 0.974 & -0.024 & -1.524 & -0.011 & -1.784 & 0 & -999 & 0 & -999 & -0.02 & -1.328 & .011 & -1.608 & 0 & -999 & 0 & -999 \\
\hline 3 & -0.005 & -0.216 & 0.009 & -1.106 & 0.128 & $2.349 *$ & 0.02 & 0.761 & -0.005 & -0.216 & -0.009 & -1.106 & 0 & -999 & 0 & -999 & 0.007 & 0.308 & -0.013 & -1.214 & 0 & -999 & 0 & -999 \\
\hline 4 & -0.022 & -0.797 & -0.025 & $-2.342 *$ & 0.15 & $2.198 *$ & 0.009 & 0.357 & -0.022 & -0.797 & \begin{tabular}{|l|}
-0.025 \\
\end{tabular} & $-2.342 *$ & 0 & -999 & 0 & -999 & $\begin{array}{l}-0.007 \\
\end{array}$ & \begin{tabular}{|l|}
-0.254 \\
\end{tabular} & $\begin{array}{ll}-0.028 \\
\end{array}$ & $-2.105^{*}$ & 0 & $\begin{array}{l}-999 \\
\end{array}$ & 0 & -999 \\
\hline 5 & -0.031 & -0.837 & -0.009 & -0.788 & 0.106 & 1.595 & 0.007 & 0.274 & -0.031 & \begin{tabular}{|l|l|}
-0.837 \\
\end{tabular} & \begin{tabular}{|l|}
-0.009 \\
\end{tabular} & -0.788 & 0 & -999 & 0 & -999 & -0.003 & -0.092 & -0.02 & -1.336 & 0 & -999 & 0 & -99 \\
\hline 6 & -0.046 & -0.99 & 0.036 & 1.946 & 0.1 & 1.573 & $\begin{array}{l}-0.007 \\
\end{array}$ & -0.394 & -0.046 & -0.99 & 0.036 & 1.946 & 0 & -999 & 0 & -999 & -0.012 & -0.266 & 19 & 1.137 & 0 & $\begin{array}{l}-999 \\
\end{array}$ & 0 & -999 \\
\hline 7 & -0.087 & -1.277 & 0.025 & 1.048 & 0.102 & 1.574 & -0.012 & -0.723 & -0.087 & -1.277 & 0.025 & 1.048 & 0 & -999 & 0 & -999 & $\begin{array}{l}-0.024 \\
\end{array}$ & -0.384 & 0.016 & 0.811 & 0 & -999 & 0 & -999 \\
\hline 8 & $\mid-0.047$ & -0.658 & 0.05 & $2.088 *$ & 0.104 & 1.575 & -0.009 & -0.674 & -0.047 & -0.658 & 0.05 & $2.088^{*}$ & 0 & -999 & 0 & -999 & 0.016 & 0.262 & 0.054 & $2.545^{*}$ & 0 & |-999 & 0 & -999 \\
\hline 9 & -0.116 & -1.201 & 0.041 & 1.202 & 0.105 & 1.569 & -0.004 & -0.607 & -0.116 & $\mid-1.201$ & 0.041 & 1.202 & 0 & -999 & 0 & $\begin{array}{l}-999 \\
\end{array}$ & \begin{tabular}{|c|}
-0.011 \\
\end{tabular} & -0.139 & 0.049 & 1.812 & 0 & -999 & 0 & -999 \\
\hline 10 & -0.186 & -1.4 & 0.08 & $4.18 *$ & 0.108 & 1.453 & 0.002 & 0.136 & -0.186 & -1.4 & 0.08 & 4.18 & 0 & -999 & 0 & -999 & 0.051 & 0.544 & 0.08 & 4.18 & 0 & -999 & 0 & -999 \\
\hline
\end{tabular}

Notes: * statistically significant at $5 \%$ level 


\section{ENDNOTES}

\footnotetext{
${ }^{1}$ Research requires the examination of all mathematical interpretations of the law. Although the functional forms of Wagner's law are similar, they are not identical. Similarity implies the existence of small differences and, since the non-linear methodology is more advanced and sensitive compared to the linear one, it grasps these differences and it may result in different degrees of causality.

${ }^{2}$ The Baek and Brock test has been applied as mentioned in Baek, E. and W. Brock (1992) and other researchers' papers (i.e. Alexakis and Siriopoulos, 1999). There have been no interventions or alterations in the test methodology.

${ }^{3}$ Non-linear causal relations have been found between variables, mainly, of the financial economics field, such as money and income (Baek and Brock, 1992), aggregate stock returns and macroeconomic factors (Hiemstra and Kramer, 1993), producer and consumer price indices (Jaditz and Jones, 1993), stock returns and volume of transactions (Hiemstra and Jones, 1992) as well as the propagation of the capital markets crisis of 1997 in Asia (Alexakis and Siriopoulos, 1999).
}

\section{AUTHOR INFORMATION}

Dr Stella Karagianni is a Professor at the Department of Economic Sciences of the University of Macedonia. She holds an MA in Economics from the Florida Atlantic University and a PhD from the University of Macedonia. She has taught Public Finance Theory and Policy, Public Administration and Policy, Public Finance History and Economic History of Greece and Macroeconomics both at the graduate and the postgraduate level. Her main research interests are in the areas of Public Finance and Environmental Economics. She has published articles in international journals and has participated in numerous research programmes and conferences.

Dr Maria Pempetzoglou studied Economics at the University of Macedonia. She holds an MPhil in Economic Development from the University of Glasgow (Centre for Development Studies) and a PhD from the University of Macedonia. She is a lecturer at the Department of Social Administration - Democritus University of Thrace. She is also a member of the Educational Staff at the Hellenic Open University and a scientific fellow at the Department of Accounting of the Technological Educational Institute of Thessaloniki. Her main research interests are in the areas of Public Finance and Environmental Economics.

\section{REFERENCES}

1. Alexakis, P. and C. Siriopoulos. The International Stock Market Crisis of 1997 and the Dynamic Relationships between Asian Stock Markets: Linear and Non-linear Granger Causality Tests, Managerial Finance, Vol. 25, No 8, pp. 22-38, 1999.

2. Baek, E. and W. Brock. A General Test for Non-linear Granger Causality: Bivariate Model, Working Paper. Iowa State University and University of Wisconsin, Madison, 1992.

3. Chletsos, M. and C. Kollias. Testing Wagner's Law using disaggregated public expenditure data in the case of Greece: 1958-1993, Applied Economics, Vol. 29, No 3, pp. 371-377, 1997.

4. Chang, T. An econometric test of Wagner's law for six countries based on cointegration and errorcorrection modelling techniques, Applied economics, Vol. 34, pp. 1157-1169, 2002.

5. Chow, Y-F., Cotsomitis, J. and A. Kwan. Multivariate Cointegration and Causality tests of Wagner's Hypothesis: evidence from the UK, Applied Economics, Vol. 34, pp. 1671-1677, 2002.

6. Denker, M. and G. Keller. On U-Statistics and Von-Mises statistics for weakly depended processes, Zeitschrift furWahrscheinlichkeitstheorie und Verwandle Gebiete, Vol. 64, pp. 505-522, 1983.

7. Dickey, A. and W.A. Fuller. Distribution of the estimators for autoregressive time series without unit root, Journal of the American Statistical Association, Vol. 74, pp. 427-431, 1979.

8. Goffman, I.. On the Empirical Testing of Wagner's Law: A Technical Note, Public Finance, Vol. 23, No. 3, p. 358, 1968.

9. $\quad$ Granger, C. W.. Forecasting in Business and Economics, Academic Press, San Diego, 1989.

10. Gupta, S.. Public Expenditure and Economic Growth: A Time Series Analysis, Public Finance, Vol. 22, No 4, pp. 423-461, 1967. 
11. Hiemstra, C. and J. D. Jones. Testing for Linear and Non-linear Granger Causality in the Stock Price Volume Relation, Journal of Finance, Vol. XLIX, No 5, pp. 1639-1664, 1994.

12. Hiemstra, C. and C. Kramer. Non-linearity and Endogeneity in macro-asset pricing, Working Paper University of Strathclyde and International Monetary Fund, IMF, 1993.

13. IMF (various issues). Yearbook of International Financial Statistics.

14. Islam, A.. Wagner's Law revisited: cointegration and exogeneity tests for the USA, Applied Economics Letters, Vol. 8, pp. 509-515, 2001.

15. Jaditz, T. and J.Jones. Granger Causality between the consumer and wholesale price indices, Working Paper Bureau of Labour Statistics and Securities and Exchange Commission, 1993.

16. Karagianni, S.. Public Sector Participation in the Development of the EU Economies, European Research Studies, Vol. 3, No 1-2, pp. 47-56, 2000.

17. Karagianni, S., Pempetzoglou, M. and S. Strikou. Linear and Non-Linear Causality Between Public Spending and Income in Greece, Political Economy, Vol. 13, pp. 57-70, 2003.

18. Karagianni, S., Pempetzoglou, M. and S. Strikou. Testing Wagner's Law for the European Union Economies, Journal of Applied Business Research, Vol. 18, No 4, pp. 107-14, 2002.

19. Loizides, J. and G., Vamvoukas. Government Expenditure and Economic Growth: Evidence from Trivariate Causality Testing, Journal of Applied Economics, Vol. 8, pp. 125-152, 2005.

20. Mann, A.. Wagner's Law: An Econometric Test for Mexico, 1925-1976, National Tax Journal, Vol. 33, No 2, p. 193, 1980.

21. Musgrave, R.A.. Fiscal Systems, Yale University Press, New Haven and London, 1969.

22. Musgrave, R.A. and P.B. Musgrave. Public Finance in Theory and Practice, McGraw-Hill, USA, 1989.

23. Osaretin, S. and T., Lorde. Co-integration, causality and Wagner's law: tests for selected Caribbean countries, Applied Economics Letters, Vol. 11, No 13, 815 - 825, 2004.

24. Peacock, A. and J., Wiseman. The Growth of Public Expenditure in the United Kingdom, Oxford University Press, London, 1961.

25. Pryor, F.. Public Expenditures in Communist and Capitalist Nations, George Allen and Unwin, London, 1968.

26. Sahni, B., S., and B. Singh. On the Causal Directions between National Income and Government Expenditure in Canada, Public Finance, Vol. 39, No 3, pp. 359-393, 1984a.

27. Singh, B. and B. S. Sahni. Causality between Public Expenditure and National Income, Review of Economics and Statistics, Vol. 66, No 4, pp. 630-643, 1984b.

28. Trotman-Dickenson, D.I.. Economics of the Public Sector, McMillan Press Ltd, London, 1996. 